\title{
Investigating the Vocabulary Load of Psychology Textbooks: A Corpus-Based Study
}

\author{
Ismail Xodabande
}

Department of Foreign Languages, Kharazmi University, No. 43, Shahid Mofatteh Street, Tehran, Iran.

Email: ismail.kh.tefl@gmail.com

ORCID ID: https://orcid.org/0000-0001-5599-8582

\begin{abstract}
:
The current corpus based study investigated the lexical profile of psychology textbooks using BNC/COCA list. To this end, 30 introductory and general psychology textbooks with around 14.8 million running words were analyzed. The findings indicated that 2048 word families provided $82.66 \%$ coverage of the corpus. The study identified 1294 high frequent and 754 midfrequent word families with high value for psychology students, which makes it possible to have a better and well-tuned vocabulary learning goals.
\end{abstract}

Keywords: lexical profile, discipline specific word lists, psychology, high-frequent words, mid-frequent words, corpus linguistics, EAP

Introduction:

Given the importance of vocabulary in developing second language knowledge in general, the field of applied linguistics has long been concerned with identifying the most useful words for language learners at different stages of learning. In a widely used classification, Coxhead and Nation (2001) divided English vocabulary into four categories: (1) high-frequency or general service vocabulary, (2) academic vocabulary, (3) technical vocabulary and (4) low-frequency vocabulary. It has been argued that beginner English language learners need to focus on the first 2000 most frequent word families of English in the General Service List (GSL) (West, 1953), while the university students should work on academic vocabulary (Coxhead, 2000; E. S.-L. Li \& Pemberton, 1994). As a result, over the past years, there has been a consistent concern among the researchers to develop academic vocabulary lists to serve the needs of language learners in university programs (Coxhead, 2000; Farrell, 1990; Gardner \& Davies, 2014; Xue \& Nation, 1984). 
Academic Word List (AWL) (Coxhead, 2000) which contains 570 word families, has been employed extensively in instructional programs (Coxhead, 2011), and remained as a benchmark for materials developments in EAP programs (e.g. Huntley, 2006; Schmitt \& Schmitt, 2005; Wells, 2007). Moreover, a number of studies have investigated its coverage in different text types in various academic disciplines (Chen \& Ge, 2007; Khani \& Tazik, 2013; Konstantakis, 2007; Y. Li \& Qian, 2010; Martínez et al., 2009; Tongpoon-Patanasorn, 2018). The overall picture provided by these studies further supported the claim that the AWL covers around 10\% of most academic texts (Coxhead, 2000; Coxhead \& Byrd, 2007), however, the studies also indicated that there is considerable variation in the coverage of the AWL among some disciplines. In line with these observations, the common core approach for identifying an academic word list to cater for the needs of students in different academic disciplines has been severely criticized (Hyland \& Tse, 2007). Moreover, by recognizing the overlap between highfrequency, academic, and technical words, a new classification system has been proposed recently, which includes high-, mid-, and low frequency vocabulary (Nation, 2013). In this new system academic and technical vocabulary are considered under the category of specialized vocabulary.

According to Coxhead (2018), the increased demand for STEM (Science, Technology, Engineering and Mathematics) education for international students has caused increased attention among the researchers to abovementioned fields. Nonetheless, she further claims that humanities have not received much attention in university vocabulary studies, and subject areas like Psychology has remained under researched. To this end, the current corpus-based study aimed to investigate the vocabulary load of psychology textbooks, and by using the classification system proposed by Nation (2013), it identifies high-, mid frequent words in psychology textbooks. The results can help both psychology students and teachers in EAP programs to set vocabulary learning goals.

\section{Method:}

The current study used a corpus of psychology e-textbooks (Appendix A). In order to make the data machine readable, the pdf files were converted to text files. The size of the corpus was 14,816,499 words. The study also used AntWordProfiler (Anthony, 2014), and the $\mathrm{BNC} / \mathrm{COCA}$ base lists to examine the corpus for its vocabulary load. The software compares the texts loaded into the program against a set of vocabulary level lists, and generate vocabulary statistic and complete frequency information about the corpus. 
In order to create high- and mid-frequency vocabulary lists for psychology textbooks, two criteria of frequency and range have been used. In this regard, following Coxhead (2000), the vocabulary items had to occur at least 28.5 times per million words in the corpus, and in at least 20 out of the 29 psychology textbooks to be included in the lists. It should be also noted that the current study used the word family defined as the base word plus its inflected forms and transparent derivations as the unit of counting.

Results and discussion:

The table 1 shows the lexical profile of psychology textbooks. The first two lists which constitute high-frequency words account for $60.88 \%$ and $11.73 \%$ of the tokens (running words) in the corpus. The cumulative coverage of these two base-list was $72.61 \%$ of the corpus. The base-list 3 to 8 that regarded as the mid-frequency words accounted for $14.03 \%$ of the tokens. Nonetheless, it should be highlighted that the third base-list alone accounted for $9 \%$ of the tokens in the corpus. Finally, the low frequent words as represented by base-list 9 to 30 accounted only for $1.6 \%$ of the corpus. The coverage of base lists 31 to 34 which include abbreviations, proper nouns was 6.85\%. Overall, the BNC/COCA base list accounted 95.09\% percent of the corpus. $4.93 \%$ of the tokens were outside the coverage of the base list, and included numbers and mostly incomplete or non-word items resulted from converting the PDF e-books to text files.

Table 1: the lexical profile of psychology textbooks based on BNC/COCA lists

\begin{tabular}{llllll}
\hline LEVEL & FILE & TOKEN & TOKEN\% & CUMTOKEN\% & GROUP \\
\hline 1 & basewrd1.txt & 9020559 & 60.88 & 60.88 & 999 \\
\hline 2 & basewrd2.txt & 1738609 & 11.73 & 72.61 & 999 \\
\hline 3 & basewrd3.txt & 1332783 & 9 & 81.61 & 1000 \\
\hline 4 & basewrd4.txt & 336590 & 2.27 & 83.88 & 994 \\
\hline 5 & basewrd5.txt & 145113 & 0.98 & 84.86 & 991 \\
\hline 6 & basewrd6.txt & 116999 & 0.79 & 85.65 & 958 \\
\hline 7 & basewrd7.txt & 77919 & 0.53 & 86.18 & 934 \\
\hline 8 & basewrd8.txt & 67924 & 0.46 & 86.64 & 898 \\
\hline 10 & basewrd9.txt & 40908 & 0.28 & 86.92 & 849 \\
\hline 11 & basewrd10.txt & 32710 & 0.22 & 87.14 & 748 \\
\hline 12 & basewrd11.txt & 30290 & 0.2 & 87.34 & 698 \\
\hline 13 & basewrd12.txt & 19168 & 0.13 & 87.47 & 569 \\
\hline 14 & basewrd13.txt & 23665 & 0.16 & 87.63 & 509 \\
\hline 15 & basewrd14.txt & 21825 & 0.15 & 87.78 & 380 \\
\hline 16 & basewrd15.txt & 14464 & 0.1 & 87.88 & 339 \\
\hline
\end{tabular}




\begin{tabular}{llllll}
\hline 17 & basewrd17.txt & 12244 & 0.08 & 88.04 & 293 \\
\hline 18 & basewrd18.txt & 6463 & 0.04 & 88.08 & 268 \\
\hline 19 & basewrd19.txt & 5970 & 0.04 & 88.12 & 219 \\
\hline 20 & basewrd20.txt & 4217 & 0.03 & 88.15 & 200 \\
\hline 21 & basewrd21.txt & 4282 & 0.03 & 88.18 & 187 \\
\hline 22 & basewrd22.txt & 2386 & 0.02 & 88.2 & 146 \\
\hline 23 & basewrd23.txt & 2748 & 0.02 & 88.22 & 142 \\
\hline 24 & basewrd24.txt & 1121 & 0.01 & 88.23 & 140 \\
\hline 25 & basewrd25.txt & 1125 & 0.01 & 88.24 & 114 \\
\hline 26 & basewrd31.txt & 471321 & 3.18 & 91.42 & 8676 \\
\hline 27 & basewrd32.txt & 403838 & 2.73 & 94.15 & 34 \\
\hline 28 & basewrd33.txt & 43537 & 0.29 & 94.44 & 589 \\
\hline 29 & basewrd34.txt & 95579 & 0.65 & 95.09 & 72047 \\
\hline 0 & - & 730501 & 4.93 & 100.02 & \\
\hline TOTAL: & 14816499 & & & 118743 & \\
\hline
\end{tabular}

In order to identify high-, mid-, and low-frequency vocabulary in psychology textbooks, the output from AntWordProfiler was copied into a Microsoft worksheet and analyzed. In this regard, 1294 word families from the two first base lists (796 in 1 and 498 in 2) met the criteria set for this study. These word families accounted for 10,611,532 tokens and $71.6 \%$ of the corpus. Comparing this coverage to total coverage provided by these lists indicated that the remaining 706 word families accounted for about $1 \%$ of the corpus. The study also identified 754 word families in base lists 3 to 8 that occurred with high frequency and acceptable range in psychology textbooks. These items accounted for 1,640,114 tokens and $11.06 \%$ of the corpus.

The findings have some implications for teaching English vocabulary for Psychology students. First, the results indicated that even in high-frequent word list, some vocabulary items tend to occur with low frequency in psychology textbooks. This is particularly important for those studying English for academic proposes in EFL context. Indeed, previous research indicated that in most EFL contexts, learners fail to acquire this essential vocabulary (Webb \& Chang, 2012). In this regard, the findings help both students and teachers to focus on the vocabulary items that are frequent in psychology texts. Second, the study also identified some midfrequency words that provide a considerable coverage of $11 \%$ in the psychology textbooks. This coverage is similar to coverage provided by AWL (Coxhead \& Byrd, 2007). Nonetheless, the AWL was created based on the GSL (West, 1953) which has been criticized for its age (Gardner \& Davies, 2014), and the use of BNC/COCA lists provide better results as their 
creation were more systematic and based on large and representative corpora. In this regard, it is possible to treat this list as an important list for psychology vocabulary. According to Nation (2013), after learning high-frequent vocabulary, language learners should proceed with focusing on mid-frequent vocabulary. As university students need to have a sufficient knowledge of these vocabulary to comprehend academic texts, teachers should provide them with appropriate materials. The findings of the current study can contribute to this need, as the list contains around 750 headwords with high value for psychology students.

\section{Conclusion:}

The current corpus based study investigated the lexical profile of psychology textbooks and created to lists of high- and mid-frequency vocabulary items to help psychology students in vocabulary learning goals. The findings revealed that 2048 word families from BNC/COCA lists provided a coverage of $82.66 \%$ in psychology textbooks. Given the enormous size of English vocabulary, this list is a valuable resource for psychology students to focus their attention and learning effort of essential vocabulary of their field. The study had some limitations that should be acknowledged. First, the corpus included only psychology textbooks, and in order to have a more comprehensive picture of vocabulary in this field, research need to investigate corpora containing other documents such as research articles and academic lectures. Second, the study was quantitative in nature and provides no information regarding the rhetorical functions of different vocabulary in psychology textbooks.

\section{References:}

Anthony, L. (2014). AntWordProfiler (Version 1.4.1) [Computer Software]. Tokyo, Japan: Waseda University. Available from https://www.laurenceanthony.net/software

Chen, Q., \& Ge, G. chun. (2007). A corpus-based lexical study on frequency and distribution of Coxhead's AWL word families in medical research articles (RAs). English for Specific Purposes, 26(4), 502-514. https://doi.org/10.1016/j.esp.2007.04.003

Coxhead, A. (2000). A New Academic Word List. TESOL Quarterly, 34(2), 213-238. https://doi.org/10.2307/3587951

Coxhead, A. (2011). The Academic Word List 10 Years On: Research and Teaching Implications. TESOL Quarterly, 45(2), 355-362. https://doi.org/10.5054/tq.2011.254528

Coxhead, A. (2018). Vocabulary and English for Specific Purposes Research. Routledge. https://doi.org/10.4324/9781315146478

Coxhead, A., \& Byrd, P. (2007). Preparing writing teachers to teach the vocabulary and grammar of academic prose. Journal of Second Language Writing, 16(3), 129-147. https://doi.org/10.1016/j.jslw.2007.07.002 
Coxhead, A., \& Nation, I. S. P. (2001). The specialised vocabulary of English for academic purposes. In J. Flowerdew \& M. Peacock (Eds.), Research Perspectives on English for Academic Purposes (pp. 252-267). Cambridge University Press. https://doi.org/10.1017/CBO9781139524766.020

Farrell, P. (1990). Vocabulary in ESP: A Lexical Analysis of the English of Electronics and a Study of Semi-Technical Vocabulary (No. 25; CLCS Occasional Paper).

Gardner, D., \& Davies, M. (2014). A New Academic Vocabulary List. Applied Linguistics, 35(3), 305-327. https://doi.org/10.1093/applin/amt015

Huntley, H. (2006). Essential Academic Vocabulary: Mastering the Complete Academic Word List. Houghton Mifflin Company.

Hyland, K., \& Tse, P. (2007). Is There an “Academic Vocabulary”? TESOL Quarterly, 41(2), 235-253. https://doi.org/10.1002/j.1545-7249.2007.tb00058.x

Khani, R., \& Tazik, K. (2013). Towards the development of an academic word list for applied linguistics research articles. RELC Journal, 44(2), 209-232. https://doi.org/10.1177/0033688213488432

Konstantakis, N. (2007). Creating a Business Word List for Teaching Business English. Estudios de Linguistica Inglesa Aplicada (ELIA), 7, 79-102.

Li, E. S.-L., \& Pemberton, R. (1994). An investigation of students' knowledge of academic and subtechnical vocabulary. Proceedings Joint Seminar on Corpus Linguistics and Lexicology, Guangzhou and Hong Kong (Language Centre, HKUST, Hong Kong, 1994), 183-196.

Li, Y., \& Qian, D. D. (2010). Profiling the Academic Word List (AWL) in a financial corpus. System, 38(3), 402-411. https://doi.org/https://doi.org/10.1016/j.system.2010.06.015

Martínez, I. A., Beck, S. C., \& Panza, C. B. (2009). Academic vocabulary in agriculture research articles: A corpus-based study. English for Specific Purposes, 28(3), 183-198. https://doi.org/10.1016/j.esp.2009.04.003

Nation, I. S. P. (2013). Learning Vocabulary in Another Language (2nd ed.). Cambridge University Press. https://doi.org/10.1017/CBO9781139858656

Schmitt, D., \& Schmitt, N. (2005). Focus on vocabulary: mastering the academic word list. Longman.

Tongpoon-Patanasorn, A. (2018). Developing a frequent technical words list for finance: A hybrid approach. English for Specific Purposes, 51, 45-54. https://doi.org/10.1016/j.esp.2018.03.002

Webb, S., \& Chang, A. C.-S. (2012). Second Language Vocabulary Growth. RELC Journal, 43(1), 113-126. https://doi.org/10.1177/0033688212439367

Wells, L. (2007). Vocabulary Mastery 1: Using and Learning the Academic Word List. University of Michigan Press.

West, M. (1953). A general service list of English words. Longman, Green \& Co.

Xue, G., \& Nation, I. S. P. (1984). A university word list. Language Learning and Communication, 3, 215-229. 
Appendix A: the books included in the corpus

1. Comer, R., \& Gould, E. (2011). Psychology Around Us. John Wiley \& Sons, Inc.

2. Coon, D., \& Mitterer, J. O. (2009). Psychology: Modules for Active Learning. Thomson Higher Education.

3. Coon, D., \& Mitterer, J. O. (2010). Introduction to Psychology. Wadsworth, Cengage Learning.

4. Coon, D., \& Mitterer, J. O. (2011). Psychology: A Journey. Wadsworth, Cengage Learning.

5. Gilbert, D., Schacter, D., \& Wegner, D. (2009). Psychology. Worth Publishers.

6. Gilbert, D., Schacter, D., \& Wegner, D. (2013). Introducing Psychology. Worth Publishers.

7. Gleitman, H. (2010). Psychology 8e. W. W. Norton \& Company, Inc.

8. Griggs, R. A. (2012). Psychology: A Concise Introduction. Worth Publishers.

9. Gross, R. (2010). Psychology: The Science of Mind and Behaviour. Hodder Education.

10. Hewstone, M., Fincham, F. D., \& Foster, J. (2005). Psychology. Blackwell Publishing Ltd.

11. Hockenbury, D. H., \& Hockenbury, S. E. (2010). Psychology. Worth Publishers.

12. Hockenbury, D. H., \& Hockenbury, S. E. (2011). Discovering Psychology. Worth Publishers.

13. Hockenbury, D. H., \& Hockenbury, S. E. (2012). Discovering Psychology. Worth Publishers.

14. Hockenbury, D. H., \& Hockenbury, S. E. (2013). Psychology. Worth Publishers.

15. Kalat, J. W. (2011). Introduction to Psychology. Wadsworth, Cengage Learning.

16. Kasschau, R. A. (2002). Understanding Psychology. McGraw-Hill.

17. Myers, D. G. (2008). Psychology in Everyday Life. Worth Publishers.

18. Myers, D. G. (2013). Psychology. Worth Publishers.

19. Myers, D. G. (2014). Exploring Psychology. Macmillan Higher Education.

20. Myers, D. G., \& Dewall, C. N. (2015). Psychology. Worth Publishers.

21. Nevid, J. S. (2009). Psychology: Concepts and Applications. Houghton Mifflin Company. 
22. Nolen-Hoeksema, S., Fredrickson, B. L., Loftus, G. R., \& Wagenaar, W. A. (2009). Atkinson \& Hilgard's Introduction to Psychology. Cengage Learning EMEA.

23. Passer, M. W., \& Smith, R. E. (2009). Psychology: The Science of Mind and Behavior. McGraw-Hill.

24. Pastorino, E., \& Doyle-Portillo, S. (2006). What Is Psychology? Thomson Higher Education.

25. Pastorino, E., \& Doyle-Portillo, S. (2013). What Is Psychology? Essentials. Wadsworth, Cengage Learning.

26. Plotnik, R., \& Kouyoumdjian, H. (2011). Introduction to Psychology. Wadsworth.

27. Rathus, S. A. (2012). Psychology: Concepts and Connections. Wadsworth Cengage Learning.

28. Schacter, D. L., Gilbert, D. T., Wegner, D. M., \& Nock, M. K. (2014). Psychology. Worth Publishers.

29. Zimbardo, P. G., Johnson, R. L., \& McCann, V. (2012). Psychology: Core Concepts. Pearson Education, Inc. 


\begin{tabular}{|c|c|c|}
\hline the & 1 & 654749 \\
\hline be & 1 & 560251 \\
\hline of & 1 & 462021 \\
\hline and & 1 & 392797 \\
\hline a & 1 & 389430 \\
\hline to & 1 & 324856 \\
\hline in & 1 & 280005 \\
\hline that & 1 & 177938 \\
\hline have & 1 & 133312 \\
\hline they & 1 & 117055 \\
\hline or & 1 & 103890 \\
\hline you & 1 & 102719 \\
\hline for & 1 & 99544 \\
\hline we & 1 & 99381 \\
\hline as & 1 & 96873 \\
\hline not & 1 & 78345 \\
\hline with & 1 & 77415 \\
\hline this & 1 & 76940 \\
\hline it & 1 & 76183 \\
\hline on & 1 & 75353 \\
\hline by & 1 & 64964 \\
\hline do & 1 & 60383 \\
\hline from & 1 & 53705 \\
\hline other & 1 & 50519 \\
\hline can & 1 & 49609 \\
\hline people & 1 & 47489 \\
\hline more & 1 & 44809 \\
\hline he & 1 & 42510 \\
\hline some & 1 & 41969 \\
\hline at & 1 & 41824 \\
\hline one & 1 & 40388 \\
\hline when & 1 & 38489 \\
\hline learn & 1 & 38393 \\
\hline i & 1 & 36961 \\
\hline brain & 2 & 36310 \\
\hline which & 1 & 36294 \\
\hline what & 1 & 36168 \\
\hline memory & 2 & 35953 \\
\hline use & 1 & 35646 \\
\hline child & 1 & 34772 \\
\hline study & 1 & 33896 \\
\hline social & 2 & 33895 \\
\hline how & 1 & 33733 \\
\hline but & 1 & 33628 \\
\hline who & 1 & 31909 \\
\hline may & 1 & 31596 \\
\hline about & 1 & 31297 \\
\hline
\end{tabular}




\begin{tabular}{|c|c|c|}
\hline think & 1 & 30528 \\
\hline develop & 2 & 30480 \\
\hline research & 2 & 29460 \\
\hline see & 1 & 29308 \\
\hline person & 1 & 29131 \\
\hline than & 1 & 28914 \\
\hline if & 1 & 28532 \\
\hline such & 1 & 28275 \\
\hline new & 1 & 27032 \\
\hline also & 1 & 26896 \\
\hline emotion & 2 & 26369 \\
\hline time & 1 & 25843 \\
\hline will & 1 & 24630 \\
\hline sex & 1 & 23959 \\
\hline she & 1 & 23936 \\
\hline group & 1 & 22979 \\
\hline make & 1 & 22740 \\
\hline chapter & 2 & 22493 \\
\hline all & 1 & 22278 \\
\hline experience & 1 & 22034 \\
\hline human & 1 & 21617 \\
\hline most & 1 & 20821 \\
\hline relate & 1 & 20773 \\
\hline test & 1 & 20632 \\
\hline self & 1 & 20488 \\
\hline process & 2 & 20304 \\
\hline science & 1 & 20282 \\
\hline many & 1 & 19505 \\
\hline example & 2 & 19227 \\
\hline figure & 1 & 19174 \\
\hline so & 1 & 19142 \\
\hline inform & 1 & 19012 \\
\hline condition & 2 & 18960 \\
\hline change & 1 & 18935 \\
\hline find & 1 & 18926 \\
\hline help & 1 & 18867 \\
\hline effect & 2 & 18770 \\
\hline two & 1 & 18380 \\
\hline work & 1 & 18204 \\
\hline feel & 1 & 17840 \\
\hline problem & 1 & 17772 \\
\hline able & 1 & 17545 \\
\hline sleep & 1 & 17524 \\
\hline system & 1 & 17306 \\
\hline mental & 2 & 17303 \\
\hline would & 1 & 17004 \\
\hline show & 1 & 16212 \\
\hline
\end{tabular}




\begin{tabular}{|c|c|c|}
\hline stress & 2 & 16197 \\
\hline high & 1 & 16032 \\
\hline out & 1 & 15889 \\
\hline between & 1 & 15854 \\
\hline culture & 2 & 15715 \\
\hline way & 1 & 15558 \\
\hline different & 1 & 15288 \\
\hline into & 1 & 15269 \\
\hline life & 1 & 15184 \\
\hline because & 1 & 15006 \\
\hline active & 2 & 14873 \\
\hline influence & 2 & 14583 \\
\hline any & 1 & 14457 \\
\hline like & 1 & 14447 \\
\hline age & 1 & 14346 \\
\hline year & 1 & 14337 \\
\hline control & 1 & 14336 \\
\hline health & 1 & 14306 \\
\hline each & 1 & 14229 \\
\hline edit & 2 & 14192 \\
\hline no & 1 & 14163 \\
\hline there & 1 & 14151 \\
\hline act & 1 & 13921 \\
\hline term & 1 & 13865 \\
\hline after & 1 & 13813 \\
\hline take & 1 & 13759 \\
\hline body & 1 & 13664 \\
\hline treat & 1 & 13509 \\
\hline part & 1 & 13502 \\
\hline woman & 1 & 13463 \\
\hline man & 1 & 13151 \\
\hline individual & 2 & 12995 \\
\hline mean & 1 & 12991 \\
\hline only & 1 & 12983 \\
\hline first & 1 & 12971 \\
\hline conscious & 2 & 12957 \\
\hline through & 1 & 12813 \\
\hline depress & 2 & 12813 \\
\hline drug & 1 & 12807 \\
\hline call & 1 & 12783 \\
\hline need & 1 & 12781 \\
\hline even & 1 & 12746 \\
\hline three & 1 & 12697 \\
\hline know & 1 & 12684 \\
\hline cause & 1 & 12603 \\
\hline become & 1 & 12498 \\
\hline associate & 2 & 12474 \\
\hline
\end{tabular}




\begin{tabular}{|c|c|c|}
\hline difference & 1 & 12437 \\
\hline adult & 2 & 12345 \\
\hline up & 1 & 12186 \\
\hline image & 2 & 12163 \\
\hline tend & 1 & 11927 \\
\hline occur & 2 & 11821 \\
\hline language & 2 & 11818 \\
\hline same & 1 & 11813 \\
\hline long & 1 & 11788 \\
\hline often & 1 & 11775 \\
\hline involve & 1 & 11774 \\
\hline include & 2 & 11723 \\
\hline event & 2 & 11707 \\
\hline then & 1 & 11671 \\
\hline question & 1 & 11669 \\
\hline page & 1 & 11620 \\
\hline result & 2 & 11596 \\
\hline during & 1 & 11565 \\
\hline likely & 2 & 11561 \\
\hline increase & 2 & 11372 \\
\hline give & 1 & 11269 \\
\hline word & 1 & 11268 \\
\hline vary & 2 & 11137 \\
\hline explain & 1 & 11111 \\
\hline nature & 1 & 11105 \\
\hline general & 1 & 10890 \\
\hline stage & 1 & 10871 \\
\hline well & 1 & 10804 \\
\hline situation & 1 & 10781 \\
\hline environme & 2 & 10781 \\
\hline form & 1 & 10732 \\
\hline parent & 1 & 10642 \\
\hline student & 1 & 10615 \\
\hline over & 1 & 10272 \\
\hline identify & 2 & 10264 \\
\hline level & 1 & 10241 \\
\hline however & 1 & 10217 \\
\hline another & 1 & 10115 \\
\hline why & 1 & 10054 \\
\hline both & 1 & 10013 \\
\hline understanc & 1 & 9879 \\
\hline early & 1 & 9868 \\
\hline could & 1 & 9854 \\
\hline observe & 2 & 9806 \\
\hline day & 1 & 9739 \\
\hline just & 1 & 9734 \\
\hline less & 1 & 9700 \\
\hline
\end{tabular}




\begin{tabular}{|c|c|c|}
\hline lead & 1 & 9623 \\
\hline mind & 1 & 9610 \\
\hline get & 1 & 9581 \\
\hline physical & 2 & 9518 \\
\hline right & 1 & 9487 \\
\hline object & 2 & 9472 \\
\hline area & 1 & 9466 \\
\hline role & 2 & 9443 \\
\hline world & 1 & 9410 \\
\hline much & 1 & 9395 \\
\hline might & 1 & 9375 \\
\hline score & 2 & 9373 \\
\hline say & 1 & 9344 \\
\hline type & 1 & 9137 \\
\hline view & 1 & 9114 \\
\hline follow & 1 & 9090 \\
\hline produce & 2 & 8999 \\
\hline late & 1 & 8958 \\
\hline move & 1 & 8957 \\
\hline measure & 2 & 8914 \\
\hline press & 1 & 8866 \\
\hline every & 1 & 8757 \\
\hline positive & 2 & 8717 \\
\hline fear & 1 & 8629 \\
\hline look & 1 & 8606 \\
\hline similar & 2 & 8598 \\
\hline important & 1 & 8556 \\
\hline perform & 2 & 8513 \\
\hline answer & 1 & 8512 \\
\hline go & 1 & 8349 \\
\hline number & 1 & 8305 \\
\hline seem & 1 & 8194 \\
\hline own & 1 & 8181 \\
\hline base & 1 & 8178 \\
\hline affect & 2 & 8127 \\
\hline approach & 2 & 8092 \\
\hline model & 2 & 8062 \\
\hline pain & 1 & 8032 \\
\hline very & 1 & 7995 \\
\hline centre & 1 & 7914 \\
\hline believe & 1 & 7904 \\
\hline begin & 1 & 7853 \\
\hline family & 1 & 7845 \\
\hline suggest & 1 & 7825 \\
\hline animal & 1 & 7757 \\
\hline case & 1 & 7737 \\
\hline dream & 1 & 7733 \\
\hline
\end{tabular}




\begin{tabular}{|c|c|c|}
\hline create & 2 & 7733 \\
\hline old & 1 & 7722 \\
\hline ask & 1 & 7705 \\
\hline set & 1 & 7640 \\
\hline while & 1 & 7631 \\
\hline university & 2 & 7566 \\
\hline eat & 1 & 7547 \\
\hline specific & 2 & 7544 \\
\hline play & 1 & 7542 \\
\hline report & 1 & 7540 \\
\hline try & 1 & 7507 \\
\hline face & 1 & 7437 \\
\hline state & 1 & 7433 \\
\hline rate & 1 & 7423 \\
\hline pattern & 2 & 7378 \\
\hline come & 1 & 7296 \\
\hline good & 1 & 7282 \\
\hline eye & 1 & 7250 \\
\hline evidence & 2 & 7232 \\
\hline reason & 1 & 7200 \\
\hline particular & 1 & 7166 \\
\hline great & 1 & 7150 \\
\hline real & 1 & 7112 \\
\hline live & 1 & 7094 \\
\hline describe & 2 & 7031 \\
\hline sense & 1 & 6921 \\
\hline before & 1 & 6891 \\
\hline point & 1 & 6873 \\
\hline light & 1 & 6866 \\
\hline school & 1 & 6745 \\
\hline although & 1 & 6733 \\
\hline express & 1 & 6727 \\
\hline normal & 1 & 6717 \\
\hline provide & 2 & 6715 \\
\hline food & 1 & 6692 \\
\hline should & 1 & 6689 \\
\hline apply & 2 & 6662 \\
\hline common & 2 & 6660 \\
\hline refer & 2 & 6647 \\
\hline usual & 1 & 6637 \\
\hline add & 1 & 6606 \\
\hline colour & 1 & 6604 \\
\hline remember & 1 & 6594 \\
\hline percent & 2 & 6576 \\
\hline read & 1 & 6546 \\
\hline sound & 1 & 6529 \\
\hline among & 1 & 6508 \\
\hline
\end{tabular}




\begin{tabular}{|c|c|c|}
\hline major & 1 & 6453 \\
\hline possible & 1 & 6453 \\
\hline young & 1 & 6450 \\
\hline reduce & 2 & 6442 \\
\hline left & 1 & 6435 \\
\hline expect & 1 & 6383 \\
\hline large & 1 & 6366 \\
\hline attention & 2 & 6361 \\
\hline attitude & 2 & 6359 \\
\hline place & 1 & 6335 \\
\hline rather & 1 & 6306 \\
\hline support & 1 & 6284 \\
\hline idea & 1 & 6267 \\
\hline simple & 1 & 6247 \\
\hline whether & 1 & 6245 \\
\hline consider & 1 & 6209 \\
\hline appear & 1 & 6191 \\
\hline friend & 1 & 6183 \\
\hline second & 1 & 6182 \\
\hline basic & 1 & 6160 \\
\hline nervous & 2 & 6138 \\
\hline must & 1 & 6083 \\
\hline male & 2 & 6079 \\
\hline actual & 1 & 6073 \\
\hline thing & 1 & 6066 \\
\hline college & 1 & 6056 \\
\hline adapt & 2 & 6035 \\
\hline rights & 1 & 5992 \\
\hline better & 1 & 5988 \\
\hline present & 1 & 5983 \\
\hline risk & 2 & 5972 \\
\hline organize & 2 & 5969 \\
\hline recall & 2 & 5910 \\
\hline without & 1 & 5887 \\
\hline certain & 1 & 5811 \\
\hline according & 2 & 5798 \\
\hline per & 2 & 5794 \\
\hline compare & 2 & 5759 \\
\hline fact & 1 & 5751 \\
\hline where & 1 & 5727 \\
\hline states & 2 & 5667 \\
\hline skill & 2 & 5657 \\
\hline now & 1 & 5656 \\
\hline drive & 1 & 5654 \\
\hline hear & 1 & 5615 \\
\hline require & 2 & 5612 \\
\hline love & 1 & 5611 \\
\hline
\end{tabular}




\begin{tabular}{|c|c|c|}
\hline react & 2 & 5569 \\
\hline value & 2 & 5502 \\
\hline few & 1 & 5478 \\
\hline key & 1 & 5467 \\
\hline goal & 2 & 5459 \\
\hline discuss & 2 & 5440 \\
\hline alcohol & 2 & 5415 \\
\hline close & 1 & 5392 \\
\hline difficult & 1 & 5391 \\
\hline practise & 2 & 5390 \\
\hline tell & 1 & 5387 \\
\hline several & 1 & 5381 \\
\hline female & 2 & 5372 \\
\hline interest & 1 & 5367 \\
\hline continue & 1 & 5273 \\
\hline represent & 2 & 5254 \\
\hline short & 1 & 5235 \\
\hline determine & 2 & 5215 \\
\hline teach & 1 & 5202 \\
\hline aware & 1 & 5196 \\
\hline turn & 1 & 5120 \\
\hline care & 1 & 5112 \\
\hline hand & 1 & 5095 \\
\hline member & 1 & 5094 \\
\hline strong & 1 & 5081 \\
\hline grow & 1 & 5059 \\
\hline classic & 2 & 4991 \\
\hline select & 2 & 4971 \\
\hline depend & 1 & 4945 \\
\hline disease & 2 & 4945 \\
\hline receive & 2 & 4944 \\
\hline want & 1 & 4943 \\
\hline toward & 1 & 4923 \\
\hline small & 1 & 4905 \\
\hline down & 1 & 4882 \\
\hline photograpl & 1 & 4850 \\
\hline success & 2 & 4845 \\
\hline mother & 1 & 4827 \\
\hline back & 1 & 4826 \\
\hline little & 1 & 4815 \\
\hline period & 2 & 4799 \\
\hline shape & 1 & 4774 \\
\hline within & 1 & 4770 \\
\hline avoid & 2 & 4726 \\
\hline train & 1 & 4705 \\
\hline four & 1 & 4699 \\
\hline order & 1 & 4680 \\
\hline
\end{tabular}




\begin{tabular}{|c|c|c|}
\hline name & 1 & 4648 \\
\hline desire & 2 & 4648 \\
\hline end & 1 & 4642 \\
\hline deep & 1 & 4621 \\
\hline profession & 2 & 4603 \\
\hline table & 1 & 4599 \\
\hline typical & 2 & 4598 \\
\hline low & 1 & 4584 \\
\hline still & 1 & 4540 \\
\hline expose & 2 & 4516 \\
\hline mood & 2 & 4508 \\
\hline easy & 1 & 4493 \\
\hline store & 1 & 4493 \\
\hline kind & 1 & 4472 \\
\hline average & 2 & 4448 \\
\hline next & 1 & 4443 \\
\hline feature & 2 & 4429 \\
\hline happy & 1 & 4419 \\
\hline nation & 1 & 4414 \\
\hline judge & 1 & 4398 \\
\hline taste & 1 & 4359 \\
\hline book & 1 & 4351 \\
\hline power & 1 & 4349 \\
\hline too & 1 & 4343 \\
\hline clear & 1 & 4336 \\
\hline note & 1 & 4333 \\
\hline rat & 2 & 4313 \\
\hline knowledge & 2 & 4293 \\
\hline month & 1 & 4283 \\
\hline allow & 1 & 4281 \\
\hline thus & 2 & 4267 \\
\hline attach & 2 & 4251 \\
\hline under & 1 & 4248 \\
\hline improve & 2 & 4246 \\
\hline especially & 1 & 4228 \\
\hline decision & 2 & 4210 \\
\hline probably & 1 & 4205 \\
\hline reserve & 2 & 4195 \\
\hline current & 2 & 4185 \\
\hline indicate & 2 & 4175 \\
\hline five & 1 & 4164 \\
\hline job & 1 & 4161 \\
\hline special & 1 & 4131 \\
\hline attract & 2 & 4130 \\
\hline cope & 2 & 4116 \\
\hline best & 1 & 4093 \\
\hline detect & 2 & 4082 \\
\hline
\end{tabular}




\begin{tabular}{|c|c|c|}
\hline signal & 2 & 4060 \\
\hline rule & 1 & 4054 \\
\hline material & 2 & 4040 \\
\hline list & 1 & 4032 \\
\hline issue & 1 & 4022 \\
\hline twin & 2 & 4022 \\
\hline side & 1 & 4015 \\
\hline hold & 1 & 4014 \\
\hline write & 1 & 3997 \\
\hline direct & 2 & 3978 \\
\hline ill & 2 & 3974 \\
\hline repeat & 2 & 3958 \\
\hline heart & 1 & 3948 \\
\hline weight & 1 & 3939 \\
\hline seek & 2 & 3932 \\
\hline course & 1 & 3917 \\
\hline loss & 2 & 3916 \\
\hline happen & 1 & 3853 \\
\hline TRUE & 1 & 3851 \\
\hline death & 1 & 3849 \\
\hline free & 1 & 3846 \\
\hline complete & 1 & 3828 \\
\hline connect & 2 & 3820 \\
\hline operate & 2 & 3813 \\
\hline wave & 1 & 3811 \\
\hline threat & 2 & 3810 \\
\hline punish & 2 & 3793 \\
\hline intense & 2 & 3788 \\
\hline society & 2 & 3772 \\
\hline educate & 1 & 3739 \\
\hline here & 1 & 3736 \\
\hline accept & 1 & 3724 \\
\hline drink & 1 & 3709 \\
\hline subject & 1 & 3706 \\
\hline hour & 1 & 3700 \\
\hline contribute & 2 & 3683 \\
\hline line & 1 & 3681 \\
\hline combine & 2 & 3678 \\
\hline let & 1 & 3665 \\
\hline programmt & 1 & 3663 \\
\hline due & 2 & 3634 \\
\hline box & 1 & 3620 \\
\hline around & 1 & 3597 \\
\hline though & 1 & 3594 \\
\hline speak & 1 & 3547 \\
\hline various & 2 & 3546 \\
\hline blood & 1 & 3534 \\
\hline
\end{tabular}




\begin{tabular}{|c|c|c|}
\hline damage & 2 & 3534 \\
\hline limit & 2 & 3513 \\
\hline white & 1 & 3506 \\
\hline energy & 2 & 3502 \\
\hline today & 1 & 3491 \\
\hline week & 1 & 3473 \\
\hline marry & 1 & 3472 \\
\hline either & 1 & 3467 \\
\hline imagine & 1 & 3461 \\
\hline unite & 2 & 3440 \\
\hline extreme & 2 & 3427 \\
\hline across & 1 & 3426 \\
\hline share & 1 & 3423 \\
\hline together & 1 & 3411 \\
\hline manage & 1 & 3409 \\
\hline correct & 2 & 3397 \\
\hline whole & 1 & 3384 \\
\hline character & 2 & 3384 \\
\hline picture & 1 & 3373 \\
\hline standard & 2 & 3368 \\
\hline discover & 1 & 3358 \\
\hline size & 1 & 3353 \\
\hline maintain & 2 & 3345 \\
\hline medical & 2 & 3345 \\
\hline lose & 1 & 3340 \\
\hline home & 1 & 3334 \\
\hline prefer & 2 & 3329 \\
\hline mark & 1 & 3321 \\
\hline lower & 2 & 3320 \\
\hline rely & 2 & 3319 \\
\hline yet & 1 & 3314 \\
\hline smoke & 1 & 3310 \\
\hline near & 1 & 3299 \\
\hline class & 1 & 3296 \\
\hline keep & 1 & 3293 \\
\hline single & 1 & 3285 \\
\hline off & 1 & 3282 \\
\hline start & 1 & 3278 \\
\hline range & 2 & 3277 \\
\hline prevent & 2 & 3255 \\
\hline history & 1 & 3254 \\
\hline motor & 2 & 3250 \\
\hline draw & 1 & 3237 \\
\hline wide & 1 & 3229 \\
\hline introduce & 2 & 3226 \\
\hline collect & 1 & 3208 \\
\hline open & 1 & 3205 \\
\hline
\end{tabular}




\begin{tabular}{|c|c|c|}
\hline exercise & 2 & 3202 \\
\hline product & 2 & 3202 \\
\hline engage & 2 & 3200 \\
\hline dog & 1 & 3190 \\
\hline forget & 1 & 3182 \\
\hline recognize & 2 & 3181 \\
\hline design & 2 & 3162 \\
\hline recent & 1 & 3154 \\
\hline amount & 1 & 3148 \\
\hline field & 1 & 3147 \\
\hline again & 1 & 3140 \\
\hline muscle & 2 & 3138 \\
\hline front & 1 & 3130 \\
\hline remove & 2 & 3130 \\
\hline section & 2 & 3129 \\
\hline once & 1 & 3125 \\
\hline concern & 1 & 3117 \\
\hline nerve & 2 & 3117 \\
\hline attempt & 2 & 3114 \\
\hline argue & 2 & 3112 \\
\hline remain & 2 & 3100 \\
\hline item & 2 & 3098 \\
\hline past & 1 & 3095 \\
\hline available & 2 & 3064 \\
\hline full & 1 & 3060 \\
\hline girl & 1 & 3057 \\
\hline baby & 1 & 3053 \\
\hline least & 1 & 3044 \\
\hline exist & 2 & 3044 \\
\hline examine & 2 & 3042 \\
\hline top & 1 & 3013 \\
\hline suffer & 2 & 2992 \\
\hline cross & 1 & 2983 \\
\hline boy & 1 & 2978 \\
\hline reach & 1 & 2971 \\
\hline far & 1 & 2968 \\
\hline pressure & 2 & 2946 \\
\hline regard & 2 & 2938 \\
\hline message & 2 & 2925 \\
\hline fall & 1 & 2924 \\
\hline detail & 2 & 2918 \\
\hline matter & 1 & 2915 \\
\hline direction & 2 & 2915 \\
\hline meet & 1 & 2913 \\
\hline company & 1 & 2906 \\
\hline contain & 2 & 2902 \\
\hline watch & 1 & 2900 \\
\hline
\end{tabular}




\begin{tabular}{|c|c|c|}
\hline separate & 2 & 2894 \\
\hline sign & 1 & 2884 \\
\hline guide & 2 & 2884 \\
\hline challenge & 2 & 2883 \\
\hline notice & 1 & 2879 \\
\hline always & 1 & 2876 \\
\hline pair & 1 & 2874 \\
\hline patient & 2 & 2866 \\
\hline responsible & 1 & 2861 \\
\hline claim & 2 & 2849 \\
\hline quick & 1 & 2846 \\
\hline never & 1 & 2834 \\
\hline step & 1 & 2829 \\
\hline talk & 1 & 2820 \\
\hline half & 1 & 2814 \\
\hline night & 1 & 2813 \\
\hline choice & 1 & 2797 \\
\hline shock & 2 & 2795 \\
\hline run & 1 & 2793 \\
\hline head & 1 & 2774 \\
\hline computer & 1 & 2773 \\
\hline speech & 2 & 2773 \\
\hline television & 1 & 2770 \\
\hline minute & 1 & 2767 \\
\hline choose & 1 & 2763 \\
\hline degree & 1 & 2756 \\
\hline locate & 2 & 2751 \\
\hline offer & 1 & 2748 \\
\hline blind & 2 & 2734 \\
\hline survive & 2 & 2733 \\
\hline middle & 1 & 2726 \\
\hline put & 1 & 2726 \\
\hline away & 1 & 2725 \\
\hline future & 2 & 2723 \\
\hline main & 1 & 2698 \\
\hline story & 1 & 2696 \\
\hline relax & 2 & 2696 \\
\hline hunger & 1 & 2689 \\
\hline perhaps & 1 & 2681 \\
\hline schedule & 2 & 2676 \\
\hline build & 1 & 2656 \\
\hline region & 2 & 2653 \\
\hline lie & 1 & 2652 \\
\hline style & 2 & 2638 \\
\hline slow & 1 & 2633 \\
\hline law & 1 & 2632 \\
\hline final & 1 & 2631 \\
\hline
\end{tabular}




\begin{tabular}{|c|c|c|}
\hline stable & 2 & 2609 \\
\hline until & 1 & 2603 \\
\hline record & 1 & 2595 \\
\hline serve & 1 & 2595 \\
\hline search & 2 & 2594 \\
\hline scale & 2 & 2571 \\
\hline attack & 2 & 2556 \\
\hline stop & 1 & 2554 \\
\hline force & 1 & 2551 \\
\hline against & 1 & 2550 \\
\hline strength & 2 & 2549 \\
\hline chance & 1 & 2546 \\
\hline medicine & 2 & 2546 \\
\hline deal & 1 & 2545 \\
\hline red & 1 & 2543 \\
\hline instead & 1 & 2538 \\
\hline almost & 1 & 2536 \\
\hline visit & 1 & 2535 \\
\hline non & 2 & 2533 \\
\hline employ & 1 & 2524 \\
\hline benefit & 2 & 2522 \\
\hline last & 1 & 2514 \\
\hline population & 2 & 2503 \\
\hline fail & 2 & 2499 \\
\hline propose & 2 & 2494 \\
\hline equal & 2 & 2485 \\
\hline birth & 1 & 2484 \\
\hline ever & 1 & 2483 \\
\hline community & 2 & 2473 \\
\hline investigate & 2 & 2468 \\
\hline walk & 1 & 2467 \\
\hline room & 1 & 2465 \\
\hline topic & 2 & 2465 \\
\hline project & 2 & 2462 \\
\hline gain & 2 & 2458 \\
\hline alter & 2 & 2447 \\
\hline quality & 2 & 2436 \\
\hline electric & 2 & 2432 \\
\hline anger & 2 & 2422 \\
\hline account & 2 & 2417 \\
\hline touch & 1 & 2416 \\
\hline spend & 1 & 2402 \\
\hline poor & 1 & 2396 \\
\hline public & 1 & 2384 \\
\hline lack & 2 & 2384 \\
\hline carry & 1 & 2369 \\
\hline ear & 1 & 2361 \\
\hline
\end{tabular}




\begin{tabular}{|c|c|c|}
\hline rapid & 2 & 2360 \\
\hline stand & 1 & 2356 \\
\hline really & 1 & 2352 \\
\hline familiar & 2 & 2349 \\
\hline smell & 1 & 2347 \\
\hline car & 1 & 2345 \\
\hline effort & 2 & 2333 \\
\hline agree & 1 & 2325 \\
\hline illustrate & 2 & 2317 \\
\hline further & 1 & 2313 \\
\hline game & 1 & 2311 \\
\hline bring & 1 & 2308 \\
\hline block & 2 & 2304 \\
\hline release & 2 & 2298 \\
\hline hard & 1 & 2293 \\
\hline instance & 2 & 2283 \\
\hline basis & 2 & 2259 \\
\hline laboratory & 2 & 2249 \\
\hline big & 1 & 2246 \\
\hline interview & 2 & 2246 \\
\hline original & 2 & 2239 \\
\hline series & 2 & 2229 \\
\hline immediate & 2 & 2228 \\
\hline return & 1 & 2225 \\
\hline assume & 2 & 2223 \\
\hline bad & 1 & 2220 \\
\hline trial & 2 & 2220 \\
\hline since & 1 & 2215 \\
\hline party & 1 & 2211 \\
\hline commit & 2 & 2211 \\
\hline brief & 2 & 2208 \\
\hline above & 1 & 2207 \\
\hline enough & 1 & 2207 \\
\hline cover & 1 & 2206 \\
\hline species & 2 & 2202 \\
\hline raise & 1 & 2195 \\
\hline leave & 1 & 2182 \\
\hline plan & 1 & 2180 \\
\hline position & 1 & 2157 \\
\hline instruct & 2 & 2149 \\
\hline pass & 1 & 2147 \\
\hline father & 1 & 2144 \\
\hline necessary & 1 & 2143 \\
\hline danger & 1 & 2140 \\
\hline video & 1 & 2140 \\
\hline pay & 1 & 2120 \\
\hline along & 1 & 2119 \\
\hline
\end{tabular}




\begin{tabular}{|c|c|c|}
\hline break & 1 & 2115 \\
\hline pleasure & 2 & 2098 \\
\hline therefore & 2 & 2093 \\
\hline alone & 1 & 2086 \\
\hline mill & 2 & 2085 \\
\hline wrong & 1 & 2082 \\
\hline green & 1 & 2076 \\
\hline black & 1 & 2070 \\
\hline below & 1 & 2068 \\
\hline music & 1 & 2067 \\
\hline send & 1 & 2066 \\
\hline previous & 2 & 2066 \\
\hline progress & 2 & 2060 \\
\hline rest & 1 & 2050 \\
\hline rise & 1 & 2046 \\
\hline brown & 1 & 2044 \\
\hline letter & 1 & 2039 \\
\hline son & 1 & 2034 \\
\hline win & 1 & 2034 \\
\hline surprise & 1 & 2022 \\
\hline die & 1 & 2017 \\
\hline panic & 2 & 2012 \\
\hline match & 2 & 1980 \\
\hline encourage & 2 & 1978 \\
\hline doctor & 1 & 1976 \\
\hline check & 1 & 1972 \\
\hline master & 1 & 1970 \\
\hline distance & 2 & 1956 \\
\hline sit & 1 & 1953 \\
\hline recover & 2 & 1953 \\
\hline beyond & 1 & 1950 \\
\hline water & 1 & 1936 \\
\hline contact & 2 & 1927 \\
\hline bottom & 1 & 1922 \\
\hline violent & 2 & 1918 \\
\hline blue & 1 & 1916 \\
\hline copy & 2 & 1915 \\
\hline sentence & 2 & 1915 \\
\hline grade & 2 & 1907 \\
\hline serious & 1 & 1904 \\
\hline van & 1 & 1904 \\
\hline exam & 2 & 1904 \\
\hline hope & 1 & 1900 \\
\hline weak & 2 & 1895 \\
\hline automatic & 2 & 1891 \\
\hline demand & 2 & 1880 \\
\hline site & 2 & 1879 \\
\hline
\end{tabular}




\begin{tabular}{|c|c|c|}
\hline prepare & 1 & 1870 \\
\hline six & 1 & 1870 \\
\hline yes & 1 & 1868 \\
\hline century & 2 & 1868 \\
\hline moment & 1 & 1862 \\
\hline decide & 1 & 1845 \\
\hline pleasant & 2 & 1843 \\
\hline sure & 1 & 1840 \\
\hline harm & 2 & 1837 \\
\hline establish & 2 & 1826 \\
\hline strange & 1 & 1807 \\
\hline aid & 2 & 1803 \\
\hline country & 1 & 1802 \\
\hline indeed & 1 & 1802 \\
\hline enter & 1 & 1800 \\
\hline couple & 1 & 1786 \\
\hline space & 1 & 1781 \\
\hline defence & 2 & 1769 \\
\hline quite & 1 & 1767 \\
\hline mate & 2 & 1765 \\
\hline resist & 2 & 1765 \\
\hline tradition & 2 & 1759 \\
\hline already & 1 & 1751 \\
\hline apparent & 1 & 1731 \\
\hline born & 1 & 1730 \\
\hline injure & 2 & 1720 \\
\hline safe & 1 & 1711 \\
\hline mathemati & 2 & 1702 \\
\hline govern & 1 & 1700 \\
\hline fast & 1 & 1689 \\
\hline tone & 2 & 1678 \\
\hline wake & 1 & 1676 \\
\hline advance & 2 & 1668 \\
\hline disturb & 2 & 1661 \\
\hline whereas & 2 & 1660 \\
\hline fire & 1 & 1647 \\
\hline protect & 1 & 1647 \\
\hline date & 1 & 1646 \\
\hline exact & 1 & 1641 \\
\hline cost & 1 & 1638 \\
\hline skin & 1 & 1624 \\
\hline else & 1 & 1622 \\
\hline victim & 2 & 1620 \\
\hline listen & 1 & 1611 \\
\hline speed & 2 & 1611 \\
\hline sad & 1 & 1610 \\
\hline divide & 2 & 1600 \\
\hline
\end{tabular}




\begin{tabular}{|c|c|c|}
\hline access & 2 & 1599 \\
\hline race & 1 & 1588 \\
\hline secure & 1 & 1588 \\
\hline directed & 2 & 1586 \\
\hline map & 2 & 1586 \\
\hline fit & 1 & 1585 \\
\hline sudden & 1 & 1582 \\
\hline million & 1 & 1581 \\
\hline balance & 2 & 1579 \\
\hline hospital & 1 & 1564 \\
\hline prison & 1 & 1563 \\
\hline popular & 2 & 1554 \\
\hline minor & 2 & 1549 \\
\hline shift & 2 & 1547 \\
\hline art & 1 & 1541 \\
\hline excite & 1 & 1541 \\
\hline telephone & 1 & 1539 \\
\hline voice & 1 & 1534 \\
\hline tense & 2 & 1534 \\
\hline opposite & 2 & 1526 \\
\hline mirror & 2 & 1517 \\
\hline suppose & 1 & 1510 \\
\hline modern & 2 & 1503 \\
\hline door & 1 & 1501 \\
\hline house & 1 & 1501 \\
\hline accident & 2 & 1498 \\
\hline diet & 2 & 1497 \\
\hline regular & 2 & 1493 \\
\hline surface & 2 & 1491 \\
\hline fat & 1 & 1486 \\
\hline foot & 1 & 1485 \\
\hline money & 1 & 1479 \\
\hline generation & 2 & 1479 \\
\hline fight & 1 & 1477 \\
\hline worth & 1 & 1476 \\
\hline cat & 1 & 1472 \\
\hline travel & 1 & 1471 \\
\hline western & 2 & 1471 \\
\hline career & 2 & 1460 \\
\hline entire & 2 & 1459 \\
\hline attend & 2 & 1457 \\
\hline hair & 1 & 1456 \\
\hline monkey & 2 & 1455 \\
\hline trouble & 1 & 1454 \\
\hline seconds & 2 & 1454 \\
\hline broad & 2 & 1453 \\
\hline bright & 1 & 1446 \\
\hline
\end{tabular}




\begin{tabular}{|c|c|c|}
\hline satisfy & 2 & 1445 \\
\hline news & 1 & 1430 \\
\hline fill & 1 & 1429 \\
\hline advertise & 1 & 1427 \\
\hline cent & 2 & 1422 \\
\hline sight & 1 & 1421 \\
\hline awake & 2 & 1421 \\
\hline print & 2 & 1420 \\
\hline tool & 2 & 1418 \\
\hline junior & 2 & 1414 \\
\hline rare & 2 & 1414 \\
\hline frustrate & 2 & 1411 \\
\hline service & 1 & 1408 \\
\hline realise & 1 & 1406 \\
\hline capable & 2 & 1397 \\
\hline nothing & 1 & 1391 \\
\hline seven & 1 & 1388 \\
\hline fix & 1 & 1387 \\
\hline ready & 1 & 1387 \\
\hline intent & 2 & 1387 \\
\hline perfect & 1 & 1382 \\
\hline lasted & 2 & 1382 \\
\hline eventually & 2 & 1374 \\
\hline total & 1 & 1365 \\
\hline purpose & 2 & 1364 \\
\hline enjoy & 1 & 1362 \\
\hline smile & 1 & 1360 \\
\hline habit & 2 & 1359 \\
\hline height & 2 & 1359 \\
\hline paper & 1 & 1351 \\
\hline street & 1 & 1341 \\
\hline team & 1 & 1337 \\
\hline obvious & 1 & 1335 \\
\hline bar & 1 & 1327 \\
\hline advantage & 2 & 1321 \\
\hline pitch & 2 & 1313 \\
\hline crime & 1 & 1310 \\
\hline ten & 1 & 1299 \\
\hline constant & 2 & 1299 \\
\hline hide & 1 & 1297 \\
\hline soon & 1 & 1291 \\
\hline sport & 1 & 1291 \\
\hline scene & 2 & 1289 \\
\hline practical & 2 & 1277 \\
\hline drama & 2 & 1267 \\
\hline economy & 2 & 1264 \\
\hline opportunit' & 2 & 1262 \\
\hline
\end{tabular}




\begin{tabular}{|c|c|c|}
\hline dark & 1 & 1258 \\
\hline fair & 1 & 1255 \\
\hline circumstan & 2 & 1254 \\
\hline kill & 1 & 1253 \\
\hline unit & 2 & 1252 \\
\hline cold & 1 & 1248 \\
\hline length & 2 & 1247 \\
\hline hall & 1 & 1246 \\
\hline post & 1 & 1246 \\
\hline pet & 2 & 1240 \\
\hline technology & 2 & 1238 \\
\hline guilty & 2 & 1232 \\
\hline worry & 1 & 1230 \\
\hline stay & 1 & 1225 \\
\hline war & 1 & 1225 \\
\hline favour & 2 & 1225 \\
\hline movie & 1 & 1218 \\
\hline flow & 2 & 1216 \\
\hline loud & 1 & 1206 \\
\hline wish & 1 & 1202 \\
\hline ground & 1 & 1201 \\
\hline bird & 1 & 1199 \\
\hline behind & 1 & 1194 \\
\hline transfer & 2 & 1191 \\
\hline air & 1 & 1189 \\
\hline inside & 1 & 1188 \\
\hline slight & 1 & 1183 \\
\hline warm & 1 & 1180 \\
\hline north & 1 & 1177 \\
\hline web & 1 & 1169 \\
\hline feed & 1 & 1168 \\
\hline film & 1 & 1167 \\
\hline wear & 1 & 1167 \\
\hline lot & 1 & 1164 \\
\hline thousand & 1 & 1164 \\
\hline screen & 2 & 1164 \\
\hline wood & 1 & 1158 \\
\hline drop & 1 & 1155 \\
\hline occasion & 2 & 1152 \\
\hline strike & 1 & 1151 \\
\hline split & 2 & 1144 \\
\hline tie & 1 & 1141 \\
\hline extend & 2 & 1141 \\
\hline respect & 2 & 1137 \\
\hline mistake & 1 & 1134 \\
\hline comfort & 1 & 1131 \\
\hline breathe & 2 & 1128 \\
\hline
\end{tabular}




\begin{tabular}{|c|c|c|}
\hline truth & 1 & 1127 \\
\hline politics & 2 & 1127 \\
\hline gay & 2 & 1126 \\
\hline bit & 1 & 1124 \\
\hline backgroune & 2 & 1119 \\
\hline miss & 1 & 1107 \\
\hline pregnant & 2 & 1105 \\
\hline bell & 2 & 1104 \\
\hline cut & 1 & 1103 \\
\hline ignore & 2 & 1101 \\
\hline mouth & 1 & 1099 \\
\hline local & 1 & 1098 \\
\hline count & 1 & 1096 \\
\hline doubt & 1 & 1092 \\
\hline concentrat & 2 & 1089 \\
\hline angry & 1 & 1083 \\
\hline address & 1 & 1079 \\
\hline city & 1 & 1075 \\
\hline sort & 1 & 1074 \\
\hline nor & 2 & 1071 \\
\hline noise & 1 & 1070 \\
\hline legal & 2 & 1064 \\
\hline wonder & 1 & 1063 \\
\hline piece & 1 & 1058 \\
\hline heavy & 1 & 1056 \\
\hline property & 2 & 1051 \\
\hline sing & 1 & 1044 \\
\hline track & 1 & 1044 \\
\hline industry & 2 & 1044 \\
\hline credit & 2 & 1042 \\
\hline tongue & 2 & 1042 \\
\hline confuse & 2 & 1041 \\
\hline root & 2 & 1040 \\
\hline arm & 1 & 1038 \\
\hline upon & 1 & 1035 \\
\hline machine & 1 & 1034 \\
\hline escape & 2 & 1034 \\
\hline fly & 1 & 1027 \\
\hline trust & 1 & 1026 \\
\hline prove & 2 & 1026 \\
\hline anxious & 2 & 1021 \\
\hline glass & 1 & 1020 \\
\hline cry & 1 & 1019 \\
\hline grey & 1 & 1018 \\
\hline bear & 1 & 1017 \\
\hline hot & 1 & 1011 \\
\hline double & 1 & 1007 \\
\hline
\end{tabular}




\begin{tabular}{|c|c|c|}
\hline famous & 2 & 1003 \\
\hline divorce & 2 & 1001 \\
\hline arrive & 1 & 999 \\
\hline card & 1 & 999 \\
\hline charge & 1 & 995 \\
\hline row & 2 & 993 \\
\hline conversatic & 1 & 992 \\
\hline circle & 2 & 988 \\
\hline september & 2 & 987 \\
\hline version & 2 & 986 \\
\hline park & 1 & 983 \\
\hline arrange & 1 & 981 \\
\hline worse & 1 & 981 \\
\hline belong & 2 & 981 \\
\hline hit & 1 & 979 \\
\hline absolute & 1 & 975 \\
\hline toy & 2 & 970 \\
\hline discipline & 2 & 967 \\
\hline opinion & 2 & 963 \\
\hline sick & 1 & 961 \\
\hline surround & 2 & 957 \\
\hline prime & 2 & 954 \\
\hline hill & 1 & 952 \\
\hline eight & 1 & 951 \\
\hline calm & 2 & 944 \\
\hline remark & 2 & 944 \\
\hline wait & 1 & 943 \\
\hline pick & 1 & 941 \\
\hline flash & 2 & 940 \\
\hline police & 1 & 937 \\
\hline bind & 2 & 936 \\
\hline closed & 1 & 933 \\
\hline edge & 1 & 933 \\
\hline ball & 1 & 932 \\
\hline bed & 1 & 931 \\
\hline replace & 2 & 931 \\
\hline branch & 2 & 922 \\
\hline witness & 2 & 920 \\
\hline king & 1 & 917 \\
\hline teenage & 2 & 915 \\
\hline trace & 2 & 910 \\
\hline fortunate & 1 & 908 \\
\hline rough & 1 & 903 \\
\hline yellow & 1 & 903 \\
\hline ordinary & 2 & 903 \\
\hline spot & 1 & 891 \\
\hline deny & 2 & 889 \\
\hline
\end{tabular}




\begin{tabular}{|c|c|c|}
\hline bond & 2 & 888 \\
\hline ago & 1 & 884 \\
\hline switch & 2 & 883 \\
\hline deliver & 2 & 881 \\
\hline morning & 1 & 879 \\
\hline flight & 2 & 878 \\
\hline egg & 1 & 876 \\
\hline twenty & 1 & 875 \\
\hline instrument & 2 & 875 \\
\hline buy & 1 & 870 \\
\hline tip & 2 & 866 \\
\hline pull & 1 & 864 \\
\hline none & 1 & 863 \\
\hline march & 2 & 861 \\
\hline business & 1 & 856 \\
\hline path & 2 & 856 \\
\hline refuse & 2 & 855 \\
\hline hundred & 1 & 854 \\
\hline mass & 2 & 853 \\
\hline forth & 2 & 852 \\
\hline smart & 2 & 849 \\
\hline finger & 1 & 847 \\
\hline apart & 1 & 846 \\
\hline neighbour & 1 & 843 \\
\hline possess & 2 & 837 \\
\hline private & 2 & 837 \\
\hline hunt & 1 & 831 \\
\hline push & 1 & 827 \\
\hline plastic & 2 & 822 \\
\hline thin & 2 & 821 \\
\hline save & 1 & 819 \\
\hline catch & 1 & 801 \\
\hline crowd & 2 & 801 \\
\hline alarm & 2 & 800 \\
\hline criminal & 2 & 799 \\
\hline assist & 2 & 794 \\
\hline stomach & 2 & 791 \\
\hline $\operatorname{mix}$ & 2 & 790 \\
\hline beauty & 1 & 789 \\
\hline gather & 2 & 789 \\
\hline wall & 1 & 786 \\
\hline nine & 1 & 784 \\
\hline cigarette & 2 & 783 \\
\hline burn & 1 & 779 \\
\hline blame & 2 & 776 \\
\hline office & 1 & 772 \\
\hline odd & 1 & 771 \\
\hline
\end{tabular}




\begin{tabular}{|c|c|c|}
\hline earn & 2 & 770 \\
\hline february & 2 & 769 \\
\hline ray & 2 & 766 \\
\hline except & 1 & 764 \\
\hline murder & 2 & 764 \\
\hline asleep & 2 & 759 \\
\hline june & 2 & 758 \\
\hline article & 2 & 757 \\
\hline stick & 1 & 756 \\
\hline sharp & 2 & 755 \\
\hline square & 1 & 748 \\
\hline nose & 1 & 742 \\
\hline wife & 1 & 741 \\
\hline shoot & 1 & 740 \\
\hline neither & 2 & 737 \\
\hline paint & 1 & 736 \\
\hline somewhat & 2 & 736 \\
\hline july & 2 & 726 \\
\hline guess & 1 & 723 \\
\hline narrow & 2 & 720 \\
\hline bridge & 2 & 719 \\
\hline frame & 2 & 719 \\
\hline library & 2 & 719 \\
\hline spread & 2 & 715 \\
\hline struggle & 2 & 715 \\
\hline remind & 2 & 713 \\
\hline snake & 2 & 711 \\
\hline nurse & 1 & 710 \\
\hline road & 1 & 706 \\
\hline sweet & 1 & 705 \\
\hline window & 1 & 703 \\
\hline oppose & 2 & 703 \\
\hline brother & 1 & 695 \\
\hline jump & 1 & 694 \\
\hline comment & 2 & 692 \\
\hline spirit & 2 & 691 \\
\hline handle & 1 & 690 \\
\hline calculate & 2 & 689 \\
\hline president & 2 & 689 \\
\hline ride & 1 & 687 \\
\hline upset & 2 & 680 \\
\hline fine & 1 & 678 \\
\hline appreciate & 2 & 678 \\
\hline departmen & 2 & 677 \\
\hline otherwise & 2 & 675 \\
\hline leg & 1 & 674 \\
\hline quiet & 1 & 674 \\
\hline
\end{tabular}




\begin{tabular}{|c|c|c|}
\hline sugar & 2 & 673 \\
\hline option & 2 & 672 \\
\hline thank & 1 & 668 \\
\hline policy & 2 & 666 \\
\hline thirst & 1 & 661 \\
\hline tree & 1 & 661 \\
\hline breast & 2 & 660 \\
\hline fun & 1 & 656 \\
\hline magazine & 2 & 655 \\
\hline sun & 1 & 650 \\
\hline warn & 2 & 647 \\
\hline price & 1 & 642 \\
\hline fright & 1 & 641 \\
\hline pound & 1 & 640 \\
\hline ship & 1 & 640 \\
\hline mention & 1 & 636 \\
\hline proper & 1 & 636 \\
\hline stroke & 2 & 636 \\
\hline quarter & 1 & 635 \\
\hline tall & 1 & 635 \\
\hline december & 2 & 635 \\
\hline convince & 2 & 634 \\
\hline november & 2 & 630 \\
\hline native & 2 & 628 \\
\hline cure & 2 & 627 \\
\hline tiny & 2 & 626 \\
\hline hurt & 1 & 624 \\
\hline lock & 1 & 623 \\
\hline ring & 1 & 623 \\
\hline roll & 1 & 623 \\
\hline meal & 1 & 620 \\
\hline sand & 2 & 620 \\
\hline afraid & 1 & 615 \\
\hline bank & 1 & 615 \\
\hline star & 1 & 615 \\
\hline straight & 1 & 615 \\
\hline disappear & 2 & 613 \\
\hline wire & 2 & 611 \\
\hline elder & 2 & 606 \\
\hline traffic & 2 & 606 \\
\hline upper & 2 & 601 \\
\hline please & 1 & 595 \\
\hline west & 1 & 594 \\
\hline chair & 1 & 592 \\
\hline ease & 2 & 592 \\
\hline relief & 2 & 592 \\
\hline unless & 1 & 589 \\
\hline
\end{tabular}




\begin{tabular}{|c|c|c|}
\hline curious & 2 & 582 \\
\hline destroy & 2 & 582 \\
\hline joy & 2 & 582 \\
\hline title & 2 & 581 \\
\hline retire & 2 & 580 \\
\hline rock & 1 & 576 \\
\hline pure & 2 & 574 \\
\hline market & 1 & 572 \\
\hline fish & 1 & 570 \\
\hline pack & 1 & 569 \\
\hline plus & 1 & 568 \\
\hline stone & 1 & 567 \\
\hline dead & 1 & 565 \\
\hline wild & 1 & 564 \\
\hline exchange & 2 & 561 \\
\hline grant & 2 & 556 \\
\hline income & 2 & 550 \\
\hline fashion & 2 & 548 \\
\hline presume & 2 & 548 \\
\hline dance & 1 & 547 \\
\hline bone & 1 & 545 \\
\hline join & 1 & 543 \\
\hline complicate & 2 & 541 \\
\hline manner & 2 & 541 \\
\hline supply & 2 & 540 \\
\hline clothes & 1 & 537 \\
\hline mouse & 2 & 537 \\
\hline forward & 1 & 536 \\
\hline billion & 1 & 534 \\
\hline daughter & 1 & 534 \\
\hline inch & 2 & 528 \\
\hline doll & 2 & 527 \\
\hline quit & 2 & 526 \\
\hline mile & 1 & 524 \\
\hline magic & 2 & 523 \\
\hline stream & 2 & 523 \\
\hline reply & 1 & 521 \\
\hline honest & 1 & 519 \\
\hline dress & 1 & 518 \\
\hline wash & 1 & 518 \\
\hline boring & 1 & 513 \\
\hline season & 2 & 513 \\
\hline land & 1 & 512 \\
\hline intend & 2 & 512 \\
\hline steady & 2 & 511 \\
\hline clue & 2 & 509 \\
\hline throw & 1 & 504 \\
\hline
\end{tabular}




\begin{tabular}{|c|c|c|}
\hline pink & 2 & 504 \\
\hline clean & 1 & 501 \\
\hline husband & 1 & 501 \\
\hline embarrass & 2 & 498 \\
\hline board & 1 & 497 \\
\hline south & 1 & 497 \\
\hline chain & 2 & 496 \\
\hline maximum & 2 & 492 \\
\hline bill & 1 & 491 \\
\hline rush & 2 & 491 \\
\hline coffee & 1 & 490 \\
\hline instant & 2 & 490 \\
\hline string & 2 & 490 \\
\hline appeal & 2 & 489 \\
\hline gold & 1 & 486 \\
\hline corner & 1 & 485 \\
\hline lay & 1 & 484 \\
\hline monday & 1 & 484 \\
\hline fold & 2 & 484 \\
\hline orange & 1 & 482 \\
\hline stare & 1 & 480 \\
\hline cliff & 2 & 480 \\
\hline salt & 2 & 479 \\
\hline empty & 1 & 477 \\
\hline finance & 2 & 476 \\
\hline admit & 1 & 474 \\
\hline guard & 2 & 469 \\
\hline recommen & 2 & 469 \\
\hline seat & 1 & 468 \\
\hline evil & 2 & 468 \\
\hline stock & 2 & 468 \\
\hline sell & 1 & 466 \\
\hline justice & 2 & 466 \\
\hline soft & 1 & 462 \\
\hline lip & 1 & 461 \\
\hline command & 2 & 461 \\
\hline blank & 2 & 460 \\
\hline engineer & 2 & 459 \\
\hline ahead & 1 & 457 \\
\hline court & 1 & 456 \\
\hline qualify & 2 & 456 \\
\hline fault & 2 & 455 \\
\hline bite & 2 & 454 \\
\hline vote & 2 & 453 \\
\hline laugh & 1 & 451 \\
\hline contract & 2 & 450 \\
\hline exhaust & 2 & 449 \\
\hline
\end{tabular}




$\begin{array}{llr}\text { mystery } & 2 & 448 \\ \text { grandfathe } & 1 & 445 \\ \text { bacon } & 2 & 445 \\ \text { file } & 2 & 445 \\ \text { beat } & 1 & 443 \\ \text { promise } & 1 & 441 \\ \text { shop } & 1 & 440 \\ \text { round } & 1 & 439 \\ \text { tempt } & 2 & 438 \\ \text { impress } & 2 & 437 \\ \text { pat } & 2 & 436 \\ \text { lesson } & 2 & 435 \\ \text { maybe } & 1 & 434 \\ \text { sister } & 1 & 434 \\ \text { suit } & 1 & 433 \\ \text { crash } & 2 & 433 \\ \text { steal } & 1 & 432 \\ \text { pretty } & 1 & 430 \\ \text { cup } & 1 & 428 \\ \text { tune } & 2 & 428 \\ \text { ice } & 1 & 427 \\ \text { faith } & 2 & 426 \\ \text { shake } & 1 & 424 \\ \text { trick } & 2 & 423 \\ \text { suck } & 2 & 422 \\ & & 10611532\end{array}$




\begin{tabular}{|c|c|c|}
\hline psychology & 3 & 77673 \\
\hline behaviour & 3 & 52518 \\
\hline disorder & 3 & 29362 \\
\hline theory & 3 & 24559 \\
\hline personality & 3 & 23346 \\
\hline et & 4 & 19900 \\
\hline therapy & 3 & 19526 \\
\hline response & 3 & 18060 \\
\hline cognitive & 4 & 16517 \\
\hline journal & 3 & 16512 \\
\hline experiment & 3 & 14307 \\
\hline stimulus & 4 & 13544 \\
\hline intelligenc€ & 3 & 13225 \\
\hline factor & 3 & 13214 \\
\hline function & 3 & 11387 \\
\hline review & 3 & 10831 \\
\hline motive & 3 & 10536 \\
\hline perception & 3 & 10271 \\
\hline neuron & 6 & 9656 \\
\hline concept & 3 & 9607 \\
\hline reinforce & 3 & 9469 \\
\hline focus & 3 & 8954 \\
\hline anxiety & 3 & 8791 \\
\hline visual & 3 & 8414 \\
\hline participant & 3 & 8293 \\
\hline cell & 3 & 8285 \\
\hline infant & 3 & 8013 \\
\hline trait & 4 & 7745 \\
\hline gender & 3 & 7662 \\
\hline define & 3 & 7413 \\
\hline genetic & 3 & 7328 \\
\hline biological & 3 & 7273 \\
\hline negative & 3 & 7041 \\
\hline content & 3 & 6921 \\
\hline structure & 3 & 6851 \\
\hline adolescent & 3 & 6827 \\
\hline schizophre। & 6 & 6821 \\
\hline task & 3 & 6801 \\
\hline sensory & 6 & 6743 \\
\hline method & 3 & 6625 \\
\hline cortex & 8 & 6584 \\
\hline perspective & 3 & 6372 \\
\hline analyse & 3 & 6344 \\
\hline psychiatry & 3 & 6243 \\
\hline predict & 3 & 6217 \\
\hline effective & 3 & 6197 \\
\hline com & 8 & 6081 \\
\hline
\end{tabular}




\begin{tabular}{|c|c|c|}
\hline symptom & 3 & 6074 \\
\hline critic & 3 & 6040 \\
\hline clinic & 3 & 6033 \\
\hline interact & 3 & 5952 \\
\hline arouse & 4 & 5907 \\
\hline perceive & 3 & 5869 \\
\hline retrieve & 4 & 5716 \\
\hline belief & 3 & 5647 \\
\hline correlate & 3 & 5514 \\
\hline gene & 3 & 5329 \\
\hline respond & 3 & 5197 \\
\hline principle & 3 & 5184 \\
\hline potential & 3 & 4985 \\
\hline technique & 3 & 4956 \\
\hline patients & 3 & 4707 \\
\hline client & 3 & 4705 \\
\hline achieve & 3 & 4630 \\
\hline stimulate & 3 & 4619 \\
\hline reflect & 3 & 4602 \\
\hline psychother & 8 & 4599 \\
\hline source & 3 & 4573 \\
\hline conflict & 3 & 4468 \\
\hline reward & 3 & 4444 \\
\hline cue & 5 & 4340 \\
\hline evolution & 3 & 4332 \\
\hline differ & 3 & 4311 \\
\hline bias & 3 & 4304 \\
\hline complex & 3 & 4301 \\
\hline solve & 3 & 4301 \\
\hline characteris & 3 & 4299 \\
\hline abuse & 3 & 4269 \\
\hline interpret & 3 & 4167 \\
\hline sensation & 4 & 4167 \\
\hline link & 3 & 4147 \\
\hline communič́ & 3 & 4119 \\
\hline assess & 3 & 4077 \\
\hline suppress & 4 & 4036 \\
\hline attribute & 3 & 4006 \\
\hline scan & 3 & 4002 \\
\hline receptor & 6 & 3975 \\
\hline activate & 4 & 3920 \\
\hline data & 3 & 3917 \\
\hline primary & 3 & 3904 \\
\hline neural & 7 & 3901 \\
\hline hypothesis & 3 & 3860 \\
\hline hormone & 4 & 3840 \\
\hline lobe & 8 & 376 \\
\hline
\end{tabular}




\begin{tabular}{|c|c|c|}
\hline aggression & 4 & 3756 \\
\hline evaluate & 3 & 3679 \\
\hline category & 3 & 3660 \\
\hline cognition & 8 & 3643 \\
\hline moral & 3 & 3579 \\
\hline relative & 3 & 3550 \\
\hline statistic & 3 & 3544 \\
\hline perceptual & 7 & 3440 \\
\hline vision & 3 & 3431 \\
\hline strategy & 3 & 3418 \\
\hline survey & 3 & 3392 \\
\hline aspect & 3 & 3387 \\
\hline sensitive & 3 & 3372 \\
\hline procedure & 3 & 3370 \\
\hline hemispherı & 5 & 3366 \\
\hline internal & 3 & 3344 \\
\hline sample & 3 & 3305 \\
\hline summary & 3 & 3301 \\
\hline abnormal & 4 & 3254 \\
\hline academy & 3 & 3220 \\
\hline aggressive & 3 & 3206 \\
\hline oriented & 3 & 3160 \\
\hline accurate & 3 & 3145 \\
\hline physiologic & 6 & 3136 \\
\hline versus & 3 & 3107 \\
\hline regulate & 3 & 3091 \\
\hline impulse & 4 & 3085 \\
\hline decrease & 3 & 3079 \\
\hline explore & 3 & 3067 \\
\hline logic & 3 & 2954 \\
\hline encode & 6 & 2926 \\
\hline demonstra & 3 & 2917 \\
\hline suicide & 3 & 2914 \\
\hline frequency & 3 & 2905 \\
\hline severe & 3 & 2892 \\
\hline significant & 3 & 2852 \\
\hline consequen & 3 & 2833 \\
\hline FALSE & 3 & 2816 \\
\hline facial & 5 & 2793 \\
\hline overall & 3 & 2780 \\
\hline mechanism & 3 & 2745 \\
\hline retina & 7 & 2730 \\
\hline distinct & 3 & 2720 \\
\hline distribute & 3 & 2715 \\
\hline outcome & 3 & 2697 \\
\hline context & 3 & 2690 \\
\hline reveal & 3 & 2682 \\
\hline
\end{tabular}




\begin{tabular}{|c|c|c|}
\hline external & 3 & 2669 \\
\hline random & 3 & 2661 \\
\hline verbal & 4 & 2642 \\
\hline enhance & 3 & 2611 \\
\hline bulletin & 5 & 2611 \\
\hline contrast & 3 & 2606 \\
\hline trauma & 4 & 2577 \\
\hline conduct & 3 & 2549 \\
\hline multiple & 3 & 2541 \\
\hline chemical & 3 & 2534 \\
\hline consistent & 3 & 2529 \\
\hline transmit & 3 & 2518 \\
\hline inhibit & 4 & 2498 \\
\hline rational & 3 & 2472 \\
\hline error & 3 & 2465 \\
\hline trigger & 3 & 2451 \\
\hline resource & 3 & 2429 \\
\hline construct & 3 & 2426 \\
\hline substance & 3 & 2384 \\
\hline capacity & 3 & 2383 \\
\hline esteem & 6 & 2376 \\
\hline text & 3 & 2346 \\
\hline insight & 3 & 2339 \\
\hline acquire & 3 & 2330 \\
\hline obey & 4 & 2327 \\
\hline restrict & 3 & 2323 \\
\hline conform & 4 & 2321 \\
\hline gland & 6 & 2317 \\
\hline frequent & 3 & 2310 \\
\hline subsequen & 3 & 2309 \\
\hline violence & 3 & 2300 \\
\hline emphasise & 3 & 2297 \\
\hline episode & 3 & 2292 \\
\hline display & 3 & 2267 \\
\hline senses & 3 & 2242 \\
\hline polar & 4 & 2241 \\
\hline media & 3 & 2215 \\
\hline publish & 3 & 2213 \\
\hline impair & 4 & 2212 \\
\hline handbook & 6 & 2211 \\
\hline copyright & 4 & 2202 \\
\hline addict & 3 & 2198 \\
\hline cerebral & 7 & 2191 \\
\hline illusion & 4 & 2185 \\
\hline psychotic & 7 & 2184 \\
\hline objective & 3 & 2170 \\
\hline averse & 7 & 2156 \\
\hline
\end{tabular}




\begin{tabular}{|c|c|c|}
\hline impact & 3 & 2148 \\
\hline description & 3 & 2131 \\
\hline component & 3 & 2130 \\
\hline failure & 3 & 2125 \\
\hline phenomen & 3 & 2119 \\
\hline behave & 3 & 2118 \\
\hline adjust & 3 & 2114 \\
\hline volume & 3 & 2105 \\
\hline mature & 3 & 2104 \\
\hline adopt & 3 & 2103 \\
\hline interfere & 3 & 2089 \\
\hline appropriatı & 3 & 2081 \\
\hline conclusion & 3 & 2071 \\
\hline promote & 3 & 2063 \\
\hline solution & 3 & 2047 \\
\hline syndrome & 4 & 2036 \\
\hline dimension & 3 & 2033 \\
\hline norm & 4 & 2029 \\
\hline cycle & 3 & 2022 \\
\hline discriminat & 3 & 2021 \\
\hline appraise & 4 & 2020 \\
\hline valid & 3 & 1990 \\
\hline independeı & 3 & 1977 \\
\hline integrate & 3 & 1971 \\
\hline schema & 7 & 1952 \\
\hline incorporate & 3 & 1945 \\
\hline audit & 3 & 1903 \\
\hline identical & 4 & 1902 \\
\hline despite & 3 & 1892 \\
\hline estimate & 3 & 1889 \\
\hline organism & 4 & 1884 \\
\hline assign & 3 & 1883 \\
\hline recognitior & 3 & 1883 \\
\hline variety & 3 & 1883 \\
\hline biology & 4 & 1881 \\
\hline status & 3 & 1871 \\
\hline spine & 4 & 1871 \\
\hline ex & 5 & 1870 \\
\hline electronic & 3 & 1862 \\
\hline medication & 5 & 1862 \\
\hline immune & 3 & 1839 \\
\hline organ & 3 & 1839 \\
\hline distress & 4 & 1820 \\
\hline implicit & 4 & 1819 \\
\hline initial & 3 & 1811 \\
\hline peer & 3 & 1794 \\
\hline importanc€ & 3 & 1793 \\
\hline
\end{tabular}




\begin{tabular}{|c|c|c|}
\hline persist & 3 & 1790 \\
\hline reflex & 6 & 1780 \\
\hline emerge & 3 & 1771 \\
\hline label & 3 & 1760 \\
\hline universe & 3 & 1748 \\
\hline alternative & 3 & 1747 \\
\hline network & 3 & 1741 \\
\hline autistic & 8 & 1738 \\
\hline archive & 4 & 1726 \\
\hline intellectual & 3 & 1724 \\
\hline chronic & 4 & 1724 \\
\hline sequence & 3 & 1720 \\
\hline ego & 4 & 1717 \\
\hline cone & 5 & 1715 \\
\hline pre & 6 & 1709 \\
\hline cord & 4 & 1706 \\
\hline mere & 3 & 1691 \\
\hline underlie & 3 & 1690 \\
\hline obsess & 4 & 1689 \\
\hline edition & 3 & 1688 \\
\hline annual & 3 & 1666 \\
\hline placebo & 7 & 1662 \\
\hline repress & 4 & 1661 \\
\hline meditate & 5 & 1656 \\
\hline code & 3 & 1654 \\
\hline counsel & 3 & 1653 \\
\hline distort & 4 & 1649 \\
\hline intervene & 3 & 1646 \\
\hline diverse & 3 & 1644 \\
\hline presence & 3 & 1638 \\
\hline induce & 4 & 1635 \\
\hline deprive & 4 & 1632 \\
\hline subjective & 4 & 1625 \\
\hline cancer & 3 & 1621 \\
\hline withdraw & 3 & 1612 \\
\hline psychoanal & 7 & 1607 \\
\hline motion & 3 & 1603 \\
\hline element & 3 & 1597 \\
\hline affection & 3 & 1591 \\
\hline unique & 3 & 1589 \\
\hline distinguish & 3 & 1585 \\
\hline implicate & 3 & 1572 \\
\hline formation & 3 & 1571 \\
\hline ethnic & 3 & 1567 \\
\hline infer & 4 & 1551 \\
\hline theme & 3 & 1547 \\
\hline imitate & 4 & 1535 \\
\hline
\end{tabular}




\begin{tabular}{|c|c|c|}
\hline nurture & 5 & 1529 \\
\hline decline & 3 & 1515 \\
\hline colleague & 3 & 1504 \\
\hline gradual & 3 & 1495 \\
\hline relevant & 3 & 1491 \\
\hline enable & 3 & 1474 \\
\hline modify & 3 & 1472 \\
\hline generate & 3 & 1469 \\
\hline instinct & 3 & 1468 \\
\hline symbol & 3 & 1467 \\
\hline homosexua & 4 & 1462 \\
\hline virtual & 3 & 1454 \\
\hline manipulat $€$ & 3 & 1443 \\
\hline psychoanal & 8 & 1443 \\
\hline threshold & 4 & 1428 \\
\hline satisfactior & 3 & 1426 \\
\hline obtain & 3 & 1419 \\
\hline disrupt & 3 & 1412 \\
\hline essential & 3 & 1410 \\
\hline rehearse & 4 & 1409 \\
\hline index & 3 & 1406 \\
\hline magnet & 3 & 1396 \\
\hline confirm & 3 & 1393 \\
\hline debate & 3 & 1393 \\
\hline excess & 3 & 1390 \\
\hline assumptior & 3 & 1386 \\
\hline temporal & 5 & 1384 \\
\hline conclude & 3 & 1378 \\
\hline hierarchy & 4 & 1370 \\
\hline input & 3 & 1364 \\
\hline hallucinate & 8 & 1361 \\
\hline monitor & 3 & 1360 \\
\hline chromoson & 6 & 1360 \\
\hline terror & 3 & 1355 \\
\hline ethical & 4 & 1349 \\
\hline reject & 3 & 1345 \\
\hline internation & 3 & 1334 \\
\hline rhythm & 3 & 1331 \\
\hline rod & 4 & 1321 \\
\hline fundament & 3 & 1320 \\
\hline cocaine & 5 & 1316 \\
\hline controvers' & 3 & 1301 \\
\hline dependenc & 4 & 1298 \\
\hline spatial & 4 & 1282 \\
\hline phase & 3 & 1280 \\
\hline consist & 3 & 1279 \\
\hline elicit & 5 & 127 \\
\hline
\end{tabular}




\begin{tabular}{|c|c|c|}
\hline molecule & 3 & 1277 \\
\hline hostile & 3 & 1275 \\
\hline intimate & 3 & 1274 \\
\hline author & 3 & 1266 \\
\hline curve & 3 & 1263 \\
\hline diagnosis & 4 & 1258 \\
\hline fluid & 4 & 1258 \\
\hline teen & 5 & 1246 \\
\hline interval & 4 & 1243 \\
\hline neutral & 3 & 1235 \\
\hline id & 5 & 1231 \\
\hline membrane & 5 & 1231 \\
\hline formal & 3 & 1230 \\
\hline origin & 3 & 1227 \\
\hline explicit & 3 & 1226 \\
\hline infect & 3 & 1223 \\
\hline target & 3 & 1215 \\
\hline ideal & 3 & 1214 \\
\hline compulsive & 8 & 1207 \\
\hline ratio & 3 & 1194 \\
\hline diagnose & 4 & 1192 \\
\hline encounter & 3 & 1186 \\
\hline mild & 3 & 1180 \\
\hline temperam & 5 & 1179 \\
\hline delay & 3 & 1177 \\
\hline chimpanzeı & 6 & 1177 \\
\hline expert & 3 & 1175 \\
\hline rear & 3 & 1165 \\
\hline majority & 3 & 1159 \\
\hline semantic & 5 & 1155 \\
\hline acquisition & 3 & 1154 \\
\hline sympathy & 3 & 1154 \\
\hline corresponc & 3 & 1144 \\
\hline permission & 3 & 1144 \\
\hline arise & 3 & 1143 \\
\hline authority & 3 & 1136 \\
\hline vulnerable & 3 & 1134 \\
\hline facilitate & 3 & 1130 \\
\hline persuasion & 5 & 1127 \\
\hline delude & 6 & 1116 \\
\hline tissue & 3 & 1106 \\
\hline periphery & 4 & 1102 \\
\hline reverse & 3 & 1087 \\
\hline predispose & 7 & 1083 \\
\hline marijuana & 7 & 1083 \\
\hline core & 3 & 1082 \\
\hline impression & 3 & 1079 \\
\hline
\end{tabular}




\begin{tabular}{|c|c|c|}
\hline efficacy & 5 & 1078 \\
\hline temperatuı & 3 & 1077 \\
\hline rape & 3 & 1072 \\
\hline extent & 3 & 1069 \\
\hline chart & 3 & 1066 \\
\hline session & 3 & 1058 \\
\hline participate & 3 & 1055 \\
\hline decade & 3 & 1053 \\
\hline device & 3 & 1053 \\
\hline empirical & 4 & 1051 \\
\hline puzzle & 3 & 1049 \\
\hline span & 4 & 1047 \\
\hline reproduce & 3 & 1045 \\
\hline contempor & 3 & 1041 \\
\hline vivid & 4 & 1041 \\
\hline graph & 4 & 1039 \\
\hline accompany & 3 & 1036 \\
\hline update & 3 & 1036 \\
\hline proceed & 3 & 1034 \\
\hline classify & 4 & 1033 \\
\hline prevalent & 4 & 1033 \\
\hline longitudina & 7 & 1033 \\
\hline religious & 3 & 1030 \\
\hline revise & 3 & 1026 \\
\hline abstract & 3 & 1022 \\
\hline disabled & 3 & 1021 \\
\hline innate & 7 & 1019 \\
\hline fulfil & 3 & 1009 \\
\hline temporary & 3 & 1008 \\
\hline physiology & 7 & 1006 \\
\hline spontaneol & 4 & 1003 \\
\hline gap & 3 & 1001 \\
\hline inherit & 3 & 992 \\
\hline deficit & 3 & 989 \\
\hline courtesy & 4 & 989 \\
\hline philosophy & 3 & 987 \\
\hline analytic & 5 & 986 \\
\hline isolate & 3 & 982 \\
\hline evolve & 3 & 972 \\
\hline genital & 7 & 969 \\
\hline alert & 3 & 967 \\
\hline surgery & 3 & 964 \\
\hline visible & 3 & 957 \\
\hline foundation & 3 & 955 \\
\hline prior & 3 & 954 \\
\hline resolve & 3 & 948 \\
\hline consume & 3 & 945 \\
\hline
\end{tabular}




\begin{tabular}{|c|c|c|}
\hline voluntary & 3 & 943 \\
\hline derive & 3 & 939 \\
\hline trend & 3 & 937 \\
\hline overcome & 3 & 935 \\
\hline division & 3 & 933 \\
\hline par & 5 & 933 \\
\hline transition & 3 & 927 \\
\hline eliminate & 3 & 925 \\
\hline transform & 3 & 925 \\
\hline cooperate & 3 & 924 \\
\hline optic & 4 & 924 \\
\hline criteria & 3 & 922 \\
\hline consult & 3 & 918 \\
\hline neurology & 7 & 915 \\
\hline exhibit & 3 & 909 \\
\hline onset & 5 & 909 \\
\hline theoretical & 3 & 906 \\
\hline approximal & 3 & 905 \\
\hline urge & 3 & 905 \\
\hline dose & 3 & 900 \\
\hline novel & 3 & 899 \\
\hline initiate & 3 & 893 \\
\hline institute & 3 & 892 \\
\hline secrete & 6 & 884 \\
\hline anorexia & 7 & 884 \\
\hline precise & 3 & 882 \\
\hline dysfunctior & 7 & 882 \\
\hline professor & 3 & 879 \\
\hline therapeutic & 5 & 879 \\
\hline request & 3 & 878 \\
\hline aim & 3 & 875 \\
\hline crisis & 3 & 875 \\
\hline athlete & 3 & 873 \\
\hline ally & 3 & 869 \\
\hline stem & 3 & 868 \\
\hline susceptible & 5 & 868 \\
\hline intelligent & 4 & 863 \\
\hline nucleus & 5 & 863 \\
\hline endure & 3 & 861 \\
\hline moderate & 3 & 861 \\
\hline vocabulary & 5 & 854 \\
\hline concrete & 3 & 851 \\
\hline prospect & 3 & 849 \\
\hline compete & 3 & 847 \\
\hline efficient & 3 & 845 \\
\hline column & 3 & 839 \\
\hline flexible & 3 & 839 \\
\hline
\end{tabular}




\begin{tabular}{|c|c|c|}
\hline overview & 5 & 824 \\
\hline distract & 4 & 821 \\
\hline coordinate & 3 & 813 \\
\hline incentive & 3 & 813 \\
\hline peak & 3 & 813 \\
\hline sibling & 5 & 813 \\
\hline confidence & 3 & 807 \\
\hline fibre & 3 & 806 \\
\hline assert & 3 & 804 \\
\hline phrase & 3 & 803 \\
\hline likeness & 3 & 798 \\
\hline cluster & 3 & 796 \\
\hline considerab & 3 & 796 \\
\hline administer & 4 & 794 \\
\hline exception & 3 & 790 \\
\hline empathy & 6 & 790 \\
\hline inject & 3 & 789 \\
\hline pose & 3 & 789 \\
\hline token & 5 & 788 \\
\hline emphasis & 3 & 786 \\
\hline dna & 3 & 784 \\
\hline latent & 6 & 783 \\
\hline companion & 3 & 782 \\
\hline ward & 4 & 780 \\
\hline diagnostic & 5 & 780 \\
\hline questionna & 4 & 776 \\
\hline superior & 3 & 775 \\
\hline maze & 6 & 775 \\
\hline accomplish & 3 & 774 \\
\hline retain & 3 & 773 \\
\hline executive & 3 & 772 \\
\hline global & 3 & 771 \\
\hline imply & 3 & 767 \\
\hline neverthele: & 3 & 766 \\
\hline conserve & 3 & 765 \\
\hline sufficient & 3 & 762 \\
\hline resemble & 3 & 761 \\
\hline mode & 3 & 758 \\
\hline recur & 4 & 758 \\
\hline destructior & 3 & 756 \\
\hline simultaneo & 3 & 754 \\
\hline ultimate & 3 & 754 \\
\hline justify & 3 & 753 \\
\hline replicate & 5 & 752 \\
\hline profile & 3 & 751 \\
\hline circuit & 3 & 750 \\
\hline former & 3 & 743 \\
\hline
\end{tabular}




\begin{tabular}{|c|c|c|}
\hline lecture & 3 & 743 \\
\hline undergo & 3 & 742 \\
\hline compreher & 4 & 742 \\
\hline nicotine & 8 & 740 \\
\hline anticipate & 3 & 737 \\
\hline inventory & 5 & 736 \\
\hline evoke & 4 & 735 \\
\hline outline & 3 & 733 \\
\hline volunteer & 3 & 732 \\
\hline cannon & 5 & 730 \\
\hline locus & 6 & 726 \\
\hline graduate & 3 & 725 \\
\hline permanent & 3 & 724 \\
\hline appetite & 5 & 722 \\
\hline sum & 3 & 721 \\
\hline oral & 3 & 720 \\
\hline route & 3 & 720 \\
\hline dent & 6 & 720 \\
\hline youth & 3 & 719 \\
\hline caffeine & 8 & 719 \\
\hline spectrum & 4 & 718 \\
\hline retard & 6 & 717 \\
\hline fetus & 8 & 717 \\
\hline approve & 3 & 715 \\
\hline myth & 3 & 715 \\
\hline intrinsic & 5 & 714 \\
\hline diminish & 4 & 711 \\
\hline physician & 4 & 710 \\
\hline criticism & 3 & 708 \\
\hline talent & 3 & 708 \\
\hline complianc€ & 4 & 701 \\
\hline dominant & 3 & 700 \\
\hline essay & 3 & 700 \\
\hline dynamic & 4 & 699 \\
\hline binge & 8 & 698 \\
\hline guideline & 3 & 696 \\
\hline expand & 3 & 695 \\
\hline foster & 3 & 686 \\
\hline convention & 3 & 684 \\
\hline emergency & 3 & 684 \\
\hline quantity & 3 & 684 \\
\hline nausea & 6 & 683 \\
\hline consistenc) & 4 & 682 \\
\hline formula & 3 & 681 \\
\hline volt & 5 & 677 \\
\hline retention & 5 & 675 \\
\hline occupation & 3 & 670 \\
\hline
\end{tabular}




\begin{tabular}{|c|c|c|}
\hline pioneer & 4 & 668 \\
\hline ambiguous & 4 & 661 \\
\hline prone & 5 & 661 \\
\hline portion & 3 & 660 \\
\hline minimise & 4 & 660 \\
\hline artificial & 4 & 658 \\
\hline disagree & 3 & 655 \\
\hline fertile & 3 & 653 \\
\hline simulate & 4 & 653 \\
\hline prescribe & 4 & 652 \\
\hline opponent & 3 & 651 \\
\hline lens & 4 & 647 \\
\hline precede & 3 & 644 \\
\hline linguistic & 4 & 644 \\
\hline hassle & 7 & 644 \\
\hline strive & 5 & 643 \\
\hline prolong & 4 & 641 \\
\hline institution & 3 & 640 \\
\hline proportion & 3 & 640 \\
\hline grammar & 5 & 640 \\
\hline glucose & 7 & 640 \\
\hline sage & 6 & 637 \\
\hline manual & 3 & 635 \\
\hline competenc & 4 & 632 \\
\hline competent & 3 & 626 \\
\hline optimist & 4 & 626 \\
\hline coefficient & 5 & 626 \\
\hline coin & 3 & 621 \\
\hline extensive & 3 & 621 \\
\hline optimal & 5 & 617 \\
\hline parallel & 3 & 613 \\
\hline gesture & 3 & 612 \\
\hline hood & 4 & 611 \\
\hline convert & 3 & 610 \\
\hline confront & 3 & 608 \\
\hline fixate & 7 & 604 \\
\hline $\operatorname{limb}$ & 4 & 601 \\
\hline virus & 3 & 599 \\
\hline attain & 4 & 599 \\
\hline restore & 3 & 598 \\
\hline heal & 3 & 594 \\
\hline numerous & 3 & 594 \\
\hline ensure & 3 & 593 \\
\hline emit & 3 & 587 \\
\hline succeed & 3 & 587 \\
\hline provoke & 3 & 584 \\
\hline corpus & 6 & 582 \\
\hline
\end{tabular}




\begin{tabular}{|c|c|c|}
\hline dual & 4 & 579 \\
\hline acute & 4 & 576 \\
\hline pill & 4 & 576 \\
\hline dot & 4 & 575 \\
\hline significanc€ & 3 & 573 \\
\hline synthesis & 4 & 571 \\
\hline ancestor & 4 & 569 \\
\hline implant & 5 & 569 \\
\hline seizure & 6 & 565 \\
\hline humour & 3 & 564 \\
\hline passive & 4 & 564 \\
\hline elevate & 3 & 563 \\
\hline highlight & 3 & 562 \\
\hline alike & 4 & 560 \\
\hline comparativ & 4 & 558 \\
\hline founded & 3 & 557 \\
\hline pupil & 3 & 557 \\
\hline differentiat & 4 & 557 \\
\hline compose & 3 & 556 \\
\hline digest & 4 & 554 \\
\hline heroin & 5 & 554 \\
\hline confident & 3 & 553 \\
\hline subtle & 3 & 553 \\
\hline mediate & 4 & 553 \\
\hline psychic & 6 & 553 \\
\hline discomfort & 7 & 548 \\
\hline yield & 3 & 547 \\
\hline reside & 3 & 541 \\
\hline serial & 5 & 540 \\
\hline trans & 8 & 539 \\
\hline deficiency & 4 & 537 \\
\hline displace & 4 & 537 \\
\hline maternal & 5 & 537 \\
\hline sperm & 6 & 536 \\
\hline disaster & 3 & 531 \\
\hline sweat & 3 & 531 \\
\hline award & 3 & 528 \\
\hline rigid & 4 & 524 \\
\hline genuine & 3 & 523 \\
\hline relatives & 3 & 523 \\
\hline fatigue & 5 & 521 \\
\hline reproducti & 5 & 520 \\
\hline dissociate & 8 & 518 \\
\hline inferior & 5 & 517 \\
\hline convey & 3 & 514 \\
\hline mammal & 4 & 514 \\
\hline mid & 4 & 513 \\
\hline
\end{tabular}




\begin{tabular}{|c|c|c|}
\hline aptitude & 8 & 513 \\
\hline terminal & 4 & 511 \\
\hline neglect & 3 & 509 \\
\hline acid & 4 & 509 \\
\hline grasp & 3 & 508 \\
\hline poverty & 3 & 507 \\
\hline boost & 3 & 506 \\
\hline fiction & 3 & 506 \\
\hline acknowled & 3 & 505 \\
\hline protein & 3 & 505 \\
\hline alternate & 4 & 502 \\
\hline diffuse & 5 & 502 \\
\hline electrode & 7 & 502 \\
\hline overwhelm & 3 & 501 \\
\hline conscientic & 7 & 501 \\
\hline elaborate & 3 & 500 \\
\hline supervise & 3 & 500 \\
\hline exaggerate & 4 & 500 \\
\hline manic & 8 & 498 \\
\hline pigeon & 5 & 496 \\
\hline rank & 3 & 495 \\
\hline resolution & 3 & 495 \\
\hline recollect & 5 & 495 \\
\hline enrich & 4 & 494 \\
\hline manifest & 3 & 493 \\
\hline exert & 4 & 493 \\
\hline spouse & 4 & 493 \\
\hline compreher & 3 & 492 \\
\hline framework & 3 & 492 \\
\hline automobile & 4 & 492 \\
\hline converge & 5 & 492 \\
\hline sane & 4 & 491 \\
\hline publication & 3 & 490 \\
\hline amplitude & 8 & 490 \\
\hline campus & 4 & 489 \\
\hline devote & 3 & 488 \\
\hline mechanic & 3 & 488 \\
\hline literal & 3 & 486 \\
\hline vast & 3 & 486 \\
\hline dilemma & 4 & 485 \\
\hline heighten & 5 & 482 \\
\hline paranoid & 7 & 480 \\
\hline feminine & 4 & 474 \\
\hline ethics & 4 & 472 \\
\hline praise & 3 & 471 \\
\hline flavour & 3 & 470 \\
\hline overlap & 4 & 470 \\
\hline
\end{tabular}




\begin{tabular}{|c|c|c|}
\hline primate & 7 & 470 \\
\hline duration & 4 & 469 \\
\hline binoculars & 5 & 467 \\
\hline contradict & 3 & 466 \\
\hline distant & 3 & 466 \\
\hline deception & 6 & 465 \\
\hline classificatic & 4 & 464 \\
\hline lateral & 5 & 464 \\
\hline fade & 3 & 461 \\
\hline mutual & 3 & 461 \\
\hline pursue & 3 & 461 \\
\hline religion & 3 & 461 \\
\hline skull & 4 & 461 \\
\hline testimony & 4 & 459 \\
\hline menstruat $\epsilon$ & 7 & 457 \\
\hline inquire & 3 & 456 \\
\hline audience & 3 & 455 \\
\hline wealth & 3 & 455 \\
\hline bizarre & 4 & 455 \\
\hline rotate & 4 & 455 \\
\hline penis & 6 & 455 \\
\hline brook & 7 & 455 \\
\hline absorb & 3 & 453 \\
\hline prize & 3 & 450 \\
\hline metabolic & 7 & 450 \\
\hline minimal & 4 & 447 \\
\hline phoneme & 8 & 447 \\
\hline tube & 3 & 445 \\
\hline contrary & 4 & 445 \\
\hline resonance & 6 & 444 \\
\hline crystal & 3 & 443 \\
\hline literature & 3 & 443 \\
\hline chemistry & 4 & 442 \\
\hline anal & 7 & 442 \\
\hline typed & 5 & 441 \\
\hline criticise & 3 & 440 \\
\hline relapse & 7 & 439 \\
\hline permanenc & 8 & 439 \\
\hline chess & 6 & 438 \\
\hline contingent & 4 & 437 \\
\hline profound & 3 & 433 \\
\hline layer & 3 & 432 \\
\hline agency & 3 & 431 \\
\hline complemeı & 4 & 427 \\
\hline independeı & 3 & 425 \\
\hline babble & 8 & 424 \\
\hline surgical & 5 & 423 \\
\hline
\end{tabular}


document

$3 \quad 422$

dynamics

422

1640114 